\title{
Les transformations d'un recueil hagiographique monumental. Le grand légendier de Saint-Maximin de Trèves aux $\mathrm{XV}^{\mathrm{e}}-\mathrm{XVI}^{\mathrm{e}}$ siècles
}

\author{
Bastien DUBUISSON \\ (Luxembourg/Namur)
}

L'abbaye bénédictine de Saint-Maximin de Trèves fut la détentrice de l'un des plus amples légendiers latins de l'époque médiévale ${ }^{1}$. Sa réalisation dans le second quart du XIII siècle précéda chronologiquement l'affirmation des Abbreviationes ou Legendae Novae/Passionalia Nova, collections hagiographiques d'un genre nouveau qui se démarquèrent notamment par leur concision et dont la Légende dorée de Jacques de Voragine constitue l'exemple le plus notoire ${ }^{2}$. En d'autres termes, la genèse de ce «grand légendier » précède le cadre temporel défini à l'occasion de ce colloque. Toutefois, considérer un légendier comme une entité statique dans le temps serait faire abstraction de données essentielles à sa bonne compréhension car l'histoire des manuscrits ne peut se borner à l'étude de leur genèse. Dans le cas du grand légendier de Trèves, l'analyse codicologique a mis en évidence les nombreuses transformations que le recueil a connu au cours des deux derniers siècles du Moyen Âge et au début des Temps modernes, une époque durant laquelle de nombreux établissements ecclésiastiques furent confrontés à un certain nombre de difficultés ayant favorisé un relâchement de la Règle et une dégradation de la vie commune ${ }^{3}$.

Il s'agira donc ici de voir comment une collection présentant encore toute les caractéristiques des légendiers dits «classiques » a évolué après l'époque de sa rédaction, suivant les besoins de ses utilisateurs et l'histoire de l'abbaye détentrice ${ }^{4}$. Après avoir

\footnotetext{
${ }^{1}$ Celui-ci a été étudié en détail dans le cadre d'un mémoire de master inédit : B. DuBuISSON, Le grand légendier de l'abbaye de Saint-Maximin de Trèves. Analyse codicologique et mise en contexte d'une collection hagiographique monumentale, Mémoire de Master en Histoire, Université catholique de Louvain, Louvain-laNeuve, 2018.

${ }^{2}$ G. PhILIPPART, Les légendiers latins et autres manuscrits hagiographiques, Turnhout, 1977 (Typologie des sources du Moyen Âge occidental, 24-25), p. 45-48. En ce qui concerne la Legenda aurea, on se réfèrera notamment aux travaux de B. Fleith, F. COSTE ou encore C. MAILlET.

${ }^{3}$ Concernant la période de «crise » traversée par les abbayes aux derniers siècles du Moyen Âge, cf., pour les établissements des Pays-Bas méridionaux, P.-J. DE GRIECK, De Benedictijnse geschiedschrijving in de Zuidelijke Nederlanden (ca. 1150-1550); historisch bewustzijn en monastieke identiteit, Leuven, 2010 (Publicaties van de Stichting Encyclopédie Bénédictine), p.92-98. En ce qui concerne Trèves, la «décadence» des abbayes bénédictines du diocèse transparaît surtout à travers les réformes menées par Jean Rode au XV siècle. À ce sujet, on renverra aux nombreuses publications de P. BECKER. La question des réformes monastiques et donc, de facto, des périodes de crises traversées par les établissements monastiques a essentiellement été traitée pour le Moyen Âge central sur lequel on a eu tendance à projeter des réalités tardo-médiévales. On pourra néanmoins renvoyer à W. SEIBRICH, «Episkopat und Klosterreform im Spätmittelalter», Römische Quartalschrift für christliche Altertumskunde und für Kirchengeschichte, 91 (1996), p. 263-338.

${ }^{4}$ On pourrait reprendre l'expression de T. SNIJDERS, Manuscript Communication. Visual and Textual Mechanics of Communication in Hagiographical Texts from the Southern Low Countries, 900-1200, Turnhout, 2015 (Utrecht Studies in Medieval Literacy, 32), p. 9, qui évoque «l'instabilité des manuscrits », une notion qui se traduit au
} 
brièvement présenté l'objet au cœur de mon enquête, j'évoquerai l'histoire des livres et de la bibliothèque de l'abbaye du $\mathrm{XIV}^{\mathrm{e}}$ au début du $\mathrm{XVI}^{\mathrm{e}}$ siècle $^{5}$. Enfin je me livrerai à une « gymnastique » codicologique et paléographique afin de mettre en évidence les interventions sur le grand légendier tout en les rattachant à leur contexte historique.

Il convient également de dire un mot sur le cadre géographique, même si celui-ci n'occupera de facto qu'un rôle mineur dans cette communication. Bien que Trèves se situe en dehors de l'espace investigué, on n'aura aucun mal à comprendre que le grand légendier de Saint-Maximin est un objet étroitement lié à ces régions limitrophes puisqu'il renferme un nombre relativement important de textes issus des diocèses voisins de Trèves, au premier rang desquels figure notamment celui de Liège ${ }^{6}$. Par ailleurs, il ne faudrait pas non plus oublier que Trèves était la métropole d'une province ecclésiastique qui englobait des diocèses situés dans l'actuel Nord-Est de la France, soit ceux de Metz, Toul et Verdun.

\section{Le grand légendier : une entreprise monumentale du XIII ${ }^{\mathrm{e}}$ siècle}

Tel qu'il nous est conservé, le grand légendier de Saint-Maximin de Trèves se présente sous la forme de huit volumes in-folio, épais d'environ 160 à 250 feuillets chacun. Un neuvième

niveau codicologique par les concepts théoriques développés par P. ANDRIST, P. CANART et M. MANIACI, $L a$ syntaxe du codex. Essai de codicologie structurale, Turnhout, 2013 (Bibliologia. Elementa ad librorum studia pertinentia, 34).

${ }_{5}$ À ce sujet, je renvoie à un autre de mes articles à paraître qui recoupera inévitablement certains des propos développés ici : B. DuBUISSON, « Bibliothéconomie et pratiques de l'écrit à Saint-Maximin de Trèves du XIII au $\mathrm{XVI}^{\mathrm{e}}$ siècle. Premiers regards sur la base du 'grand légendier' et d'autres manuscrits hagiographiques », Questes. Revue pluridisciplinaire d'études médiévales.

${ }^{6}$ Le découpage « géographique » du sanctoral du grand légendier, partant du centre (Trèves) pour aller vers les périphéries, montre un espace qui, dans ses grandes lignes, correspond à l'ancienne Lotharingie. Le cœur de la matière hagiographique, soit le contingent des saints « régionaux » est essentiellement fourni par les diocèses de Trèves, Liège et Cologne. Sur l'espace lotharingien, cf. M. MARGUE, «Lotharingien als Reformraum (10. bis Anfang des 12. Jahrhunderts). Einige einleitende Bemerkungen zum Gebrauch räumlicher und religiöser Kategorien », dans Lotharingien und das Papsttum im Früh- und Hochmittelalter. Wechselwirkungen im Grenzraum zwischen Germania und Gallia, éd. K. HERBERS, H. MüLlER, Berlin-Boston, 2017 (Abhandlungen der Akademie der Wissenschaften zu Göttingen; Neue Folge, 45 : Studien zu Papstgeschichte und Papsturkunden), p. 14-22 ; M. PARISSE, Religieux et religieuses en Empire du $X^{e}$ au XII siècle, Paris, 2011 (Les médiévistes français, 11), p. 32-37. Pour ce qui est des saints lotharingiens, on renverra à T. BAUER, Lotharingien als historischer Raumbildung und Raumbewußtsein im Mittelalter, Cologne et al., 1997 (Rheinisches Archiv, 136), qui s'est justement appuyé sur des sources du Moyen Âge central et du bas Moyen Âge pour délimiter un contingent régional. On lui a notamment reproché de projeter ces réalités sur le haut Moyen Âge : J. SCHNEIDER, Auf der Suche nach dem verlorenen Reich. Lotharingien im 9. und 10. jahrhundert, Cologne et al., 2010 (Publications du Centre Luxembourgeois de Documentation et d'Études Médiévales, 30), p. 217-220. Voir depuis T. BAUER, "Gibt es lotharingische Heiligen », dans La Lotharingie en question. Identités, oppositions, intégration. Actes des 14es Journées Lotharingiennes, Luxembourg, 2018 (Publication de la Section Historique de l'Institut Grand-Ducal, 126 ; Publications du CLUDEM, 26), p. 421-456. 
volume contenant le sanctoral relatif au mois de décembre a disparu ${ }^{7}$. Cet ensemble organisé per circulum anni couvrait donc l'entièreté de l'année liturgique ${ }^{8}$.

Qualifié dès la fin du XIX ${ }^{\mathrm{e}}$ siècle, de « grand légendier »(Das große Trierer Legendar $)^{9}$, la collection de Saint-Maximin ne se démarque pourtant pas en raison de son format ${ }^{10}$ : ses volumes figurent dans la norme des manuscrits médiévaux et se classent même loin derrière les légendiers italiens du XII ${ }^{\mathrm{e}}$ siècle par exemple ${ }^{11}$. C'est donc bien par le biais de la densité de son sanctoral que le légendier de Saint-Maximin peut être qualifié de « grand » puisque la collection regroupait à l'origine plus de 400 dossiers hagiographiques, ce qui en fait l'un des ultimes témoins de l'accroissement du sanctoral des recueils hagiographiques occidentaux ainsi que l'un des plus amples légendiers latins du Moyen Âge ${ }^{12}$. À ce titre, il fait partie du cercle restreint

${ }^{7}$ Lorsque deux bénédictins de la congrégation de Saint-Maur visitèrent l'abbaye en 1718 , la collection ne comptait
déjà plus que huit volumes : «Il y a aussi un très beau recueil de vies de Saints distribuées par mois, en huit gros
volumes, d'une écriture d'environ six cens ans ». E. MARTENE et U. DURAND, Voyage littéraire de deux religieux
bénédictins de la congrégation de S. Maur, vol. 2, Paris, 1724, p. 285. La disparition du volume serait même
antérieure à l'entreprise bollandiste ainsi qu'à ses prémices initiées par Héribert Rosweyde (1569-1629). De fait,
on n'en a pas trace dans les Collectanea Bollandiana, ces volumes préparatoires aux Acta Sanctorum,
contrairement aux huit autres volumes de la collection. Pourtant, Rosweyde s'était déjà employé, entre 1607 et
1629 , à amasser maintes copies de manuscrits, dont certains exemplaires étaient issus de Saint-Maximin :
F. DolBEAU, "Les sources manuscrites des Acta Sanctorum et leur collecte (XVII-XVIII siècles)», dans De
Rosweyde aux Acta Sanctorum. La recherche hagiographique des Bollandistes à travers quatre siècles. Actes du
Colloque international (Bruxelles, 5 octobre 2007), éd. R. GoDDING et al., Bruxelles, 2009 (Subsidia hagiographica, 88), p. 118-119.

${ }^{8}$ Paris, Bibliothèque nationale de France, lat. 9741 (janvier) ; Trèves, Stadtbibliothek, 1151/453 (février-avril); 1151/454 (mai-juin) ; 1151/455 (juin-juillet) ; Paris, Bibliothèque nationale de France, lat. 9742 (août) ; Trèves, Bibliothek des Bischöflichen Priesterseminars, 35 (septembre); Trèves, Stadtbibliothek, 1151/456 (octobre); Trèves, Bibliothek des Bischöflichen Priesterseminars, 36 (novembre).

${ }^{9}$ L'expression remonte à B. KRUSCH, « Reise nach Frankreich im Frühjahr und Sommer 1892 », Neues Archiv der Gesellschaft für ältere deutsche Geschichtskunde, 18 (1893), p. 618.

${ }^{10}$ Les huit volumes conservés, abstraction faite des quelques millimètres de rognage, sont tous de dimensions similaires, à savoir, en moyenne, $33,33 \times 23,24 \mathrm{~cm}$. En additionnant la somme de la hauteur et de la largeur de chaque manuscrit, puis en calculant la moyenne, on obtient $565,63 \mathrm{~mm}$, ce qui permet de classer les volumes du grand légendier parmi les livres de format «moyen-grand »: C. BOzzolo et E. ORNATO, Pour une histoire du livre manuscrit au Moyen Âge. Trois essais de codicologie quantitative, Paris, 1983 (Équipe de recherche sur l'humanisme français ; Textes et études, 2), p. 218 ; quant à leurs proportions (sur la base du rapport de la largeur divisée par la hauteur), elles se rapprochent de la constante définie pour un échantillon de manuscrits allant du $\mathrm{VIII}^{\mathrm{e}}$ au XIII ${ }^{\mathrm{e}}$ siècle, en se situant entre 0,60 et 0,80 , voire même entre 0,67 et 0,72 pour la grande majorité des cas : J. P. GuMBerT, «The Sizes of Manuscripts. Some Statistics and Notes », dans Hellinga Festschrift. Forty-three Studies in Bibliography presented to Prof. Dr. Wytze Hellinga on the occasion of his retirement from the Chair of Neophilology in the University of Amsterdam at the end of the year 1978, éd. A. R. A. CROISET VAN UCHELEN, Amsterdam, 1980, p. 285.

${ }^{11}$ G. Philippart, Les légendiers latins, p. 37-38, a dressé un tableau comparatif des dimensions des légendiers latins contenant la Passio Cypriani. Le grand légendier de Saint-Maximin, représenté par son volume du mois de septembre (Trèves, Bibliothek des Bischöflichen Priestrseminars, 35), se situe en effet en-deçà des trois quarts des manuscrits pris en compte par l'auteur, qui mesurent entre 350 et $470 \mathrm{~mm}$ et est à ce titre classé parmi les manuscrits dits « plus petits ». De la même manière, le grand légendier ne se plie pas aux observations concernant l'évolution des légendiers des Pays-Bas méridionaux qui, à partir du XII ${ }^{\mathrm{e}}$ siècle, «mesuraient d'habitude plus de $40 \times 25 \mathrm{~cm} »$. T. SNIJDERS, Manuscript Communication, p. 366 .

${ }^{12}$ G. PhilipPart, Les légendiers latins, p. 39-40, présente un second tableau comparatif relatif cette fois à l'ampleur du sanctoral des légendiers latins contenant la Passio Cypriani. Pour la "période classique » (IX ${ }^{\mathrm{e}}-\mathrm{XIII}{ }^{\mathrm{e}}$ siècle), le grand légendier de Saint-Maximin n'est dépassé de justesse que par deux exemplaires du grand légendier d'Autriche (fin XII ${ }^{\mathrm{e}}$-début XIII ${ }^{\mathrm{e}}$ siècle). Si l'on se limite strictement à la région rhéno-mosane, il faudra attendre le 
de ces amples recueils auxquels on a attribué une appellation spécifique : il s'agit des « grands légendiers » (Magna legendaria) d'Autriche et de Flandre ou encore du Liber de Natalitiis, trois collections constituées au tournant des $\mathrm{XII}^{\mathrm{e}}-\mathrm{XIII}{ }^{\mathrm{e}}$ siècles, dont la diffusion s'est concentrée dans une aire géographique spécifique avec une présence toute particulière en milieu cistercien. Toutefois, contrairement au « grand légendier de Trèves », dénomination qui ne concerne que l'unique exemplaire maximinien ${ }^{13}$, ces collections sont connues et ont été reconstituées à travers une série de légendiers témoins souvent incomplets ${ }^{14}$.

Chronologiquement parlant, l'historiographie attribue au grand légendier le terminus post quem de 1235, date de la canonisation d'Élisabeth de Hongrie dont le dossier figure dans le volume de novembre (BHL 2493 et 2509$)^{15}$. Mes investigations ont permis de confirmer que le grand légendier avait bel et bien été produit dans le courant des années 1230-1240, soit sous les abbatiats respectifs de Bartholomé d'Esch et de Henri III de Bruch ${ }^{16}$, à un moment charnière de l'histoire de l'ancienne abbaye impériale. De fait, d'après l'historiographie, Saint-Maximin

$\mathrm{XV}^{\mathrm{e}}$ siècle pour que la collection de Jean Gielemans (1470) de l'abbaye de Rouge-Cloître dépasse en ampleur le grand légendier de Saint-Maximin. Il faut toutefois noter que cette collection constitue un exemple de légendier latin tardif tout à fait exceptionnel. Ibid., p. 37. Sur Jean Gielemans, cf. V. Souche-HAZEBroucK, Spiritualité, sainteté et patriotisme : glorification du Brabant dans l'œuvre hagiographique de Jean Gielemans (1427-1487), Turnhout, 2007 (Hagiologia, 6).

${ }^{13}$ L'abbaye bénédictine voisine de Saint-Euchaire/Matthias a pu posséder un voire deux grand(s) légendier(s). On conserve en effet deux volumes (Trèves, Stadtbibliothek, 1152/776 et Bibliothek des Bischöflichen Priesterseminars, 5) du XII ${ }^{\mathrm{e}}$ siècle qui partagent un programme hagiographique très proche de celui du légendier de Saint-Maximin, bien que ce dernier ait été amplifié.

${ }^{14}$ G. Philippart, Les légendiers latins, p. 37-40. La collection connue sous le nom de Magnum Legendarium Austriacum est née à la fin du $\mathrm{XII}^{\mathrm{e}}$ siècle et s'est diffusée jusqu'au $\mathrm{XV}^{\mathrm{e}}$ siècle dans une série d'établissements monastiques, principalement cisterciens, situés sur le territoire de l'actuelle Autriche : D. Ó RIAIN, « The Magnum Legendarium Austriacum : a new investigation of one of medieval Europe's richest hagiographical collections "), Analecta Bollandiana, 133 (2015), p. 99-106; A. KERN, «Magnum Legendarium Austriacum », dans Die Österreichische Nationalbibliothek. Festschrift zum 25-jährigen Dienstjubiläum des Generaldirektors Univ.-Prof. Dr. Josef Bick, éd. J. STummVolL, Vienne, 1948, p. 429-434. De son côté, le Legendarium Flandrense constitue une collection d'origine cistercienne née au début du XIII ${ }^{\mathrm{e}}$ siècle en Flandre maritime, dont une version plus ample s'est ensuite répandue dans le Cambrésis, le Hainaut et l'Artois : S. LECOUTEUX, «Les anciens légendiers de Cambrai $\left(\mathrm{X}^{\mathrm{e}}-\mathrm{XII}^{\mathrm{e}} \mathrm{s}\right.$.) : structures et parenté avec le 'grand légendier de Flandre' (Legendarium Flandrense) », dans Le livre et l'écrit : texte, liturgie et mémoire dans l'Église au Moyen Âge, éd. J.-L. DeufFIC, Turnhout, 2012 (Pecia, 14), p. 109-184 ; F. DolbEAU, " Nouvelles recherches sur le 'Legendarium Flandrense' », Recherches augustiniennes, 16 (1981), p. 399-455. Enfin, le Liber de Natalitiis est une collection en six volumes qui fut majoritairement diffusée dans les abbayes cisterciennes du Nord de la France entre la fin du XII et le XIV ${ }^{\mathrm{e}}$ siècle. F. Dolbeau, « Notes sur la genèse et sur la diffusion du Liber de natalitiis », Revue d'histoire des textes, vol. 6, 1976 (1978), p. 143-195 ; H. RochAIS, Liber de natalitiis et autres légendiers du Moyen Âge, 3 vol., Rochefort, 1975 (Documentation cistercienne, 15).

15 Trèves, Bibliothek des Bischöflichen Priesterseminars, 36, fol. 92r-99v. À propos d'Élisabeth de Thuringe, cf. R. Folz, Les saintes reines du Moyen Âge en Occident (VI ${ }^{e}-X I I I^{e}$ siècles), Bruxelles, 1992 (Subsidia hagiographica, 76), p. 105-129.

${ }^{16}$ Sur ces deux abbés, cf. Die Benediktinerabtei St. Maximin vor Trier, éd. B. REsmini, vol. 2, Berlin/Boston, 2016 (Germania Sacra; Dritte Folge, 11 ; Das Erzbistum Trier, 13), p. 1066-1069. Ils sont tous deux mentionnés dans l'obituaire du livre du chapitre (Trèves, Stadtbibliothek, 1634/394, fol. 139v-151r), respectivement le 28 février (fil. 141r : "Henricus, abbas hujus loci, fundator hospitalis sanctae Elysabeth ») et le 14 novembre (fol. 150r : «Bartholomeus abbas hujus loci, qui plura beneficia fecit fratribus »). 
aurait connu, après sa soumission à l'archevêque de Trèves en $1139^{17}$, un important déclin ayant eu des conséquences néfastes sur l'activité de son scriptorium ${ }^{18}$, ce qui poussait notamment

Anton Chroust à considérer le grand légendier comme une production de l'abbaye voisine de Saint-Matthias ${ }^{19}$.

Pourtant, dans la première moitié du XIII ${ }^{\mathrm{e}}$ siècle, un concours de circonstances permit à l'abbaye de retrouver un certain dynamisme ${ }^{20}$. En 1231, on assista notamment à la consécration

${ }^{17}$ K. KRÖNERT, «Bernard de Clairvaux et l'affaire de Saint-Maximin de Trèves : entre idéaux réformateurs et relations amicales », Revue du Nord, 391-392 (2011), p. 779-794 ; J. R. MÜLLER, Vir religiosus ac strenuus. Albero von Montreuil, Erzbischof von Trier (1132-1152), Trèves, 2006 (Trierer historische Forschungen, 56), p. 387-420 et 625-658 ; H. BÜTTNER, « Der Übergang der Abtei St. Maximin an das Erzstift Trier unter Erzbischof Albero von Montreuil», dans Festschrift Ludwig Petry, éd. J. BÄrmann, K. G. Faber, A. Gerlich, vol. 1, Wiesbaden, 1968 (Geschichtliche Landeskunde, 5), p. 65-77.

${ }^{18}$ Die Benediktinerabtei, vol. 1, p. 273-277. En réalité, le devenir des abbayes est relativement peu connu en ce qui concerne la seconde moitié du XII ${ }^{\mathrm{e}}$ siècle. Certains établissements, tels que Stavelot, Gembloux, Saint-Trond ou encore Saint-Hubert, connurent d'importants problèmes financiers. De manière générale, la littérature scientifique a mis en exergue l'important déclin spirituel ayant frappé les abbayes bénédictines au tournant des $\mathrm{XII}^{\mathrm{e}}$ et $\mathrm{XIII}^{\mathrm{e}}$ siècles: T. SNIJDERS, Manuscript Communication, p. 35 ; P.-J. DE GRIECK, De benedictijnse geschiedschrijving, p. 74 sqq.

${ }^{19}$ D'après F. AVRIL et C. RABEL, Manuscrits enluminés d'origine germanique, vol. 1, Paris, 1995, p. 148. Comme noté plus haut, cette abbaye bénédictine voisine possédait depuis le XII ${ }^{\mathrm{e}}$ siècle au moins deux légendiers partageant une partie significative du programme du légendier de Saint-Maximin, du moins en ce qui concerne les mois de janvier à mars : cf. n. 13. Or, comme le soulignait G. PHILIPPART, Les légendiers latins, p. 101, les collections hagiographiques latines se seraient diffusées à la manière de la «boule de neige » : un tronc commun aurait été copié d'un recueil à un autre tout en étant continuellement augmenté par l'ajout de textes locaux ou à faible diffusion. Que ce tronc commun inclue les deux légendiers de Saint-Matthias n'aurait rien de surprenant : outre la proximité géographique, deux abbés de Saint-Matthias furent abbés d'Echternach avant Bartholomé d'Esch. Les collections hagiographiques du tournant des $\mathrm{XII}^{\mathrm{e}}$-XIII ${ }^{\mathrm{e}}$ siècles de ces trois abbayes bénédictines - Saint-Maximin, Saint-Matthias et Saint-Willibrord d'Echternach - présentent donc nécessairement des similitudes. Dès lors, on ne pourra pas étudier l'un de ces légendiers sans prendre en compte les autres de même que l'intégralité du « patrimoine hagiographique » de ces trois établissements.

${ }^{20} \hat{A}$ partir du second quart du XIII ${ }^{\mathrm{e}}$ siècle, une entente cordiale et une étroite collaboration entre l'abbaye et les archevêques de Trèves fut initiée, tant au niveau religieux que politique : B. RESMINI, « Adler und Bär. Aspekte zur verfassungsmäßigen Stellung der Abtei St. Maximin im späten Mittelalter und in der frühen Neuzeit», Jahrbuch für westdeutsche Landesgeschichte, 30 (2004), p. 38. Lors de l'absence du métropolitain, il revenait à l'abbé de Saint-Maximin le droit d'exercer certains pouvoirs épiscopaux, notamment lors de la procession du dimanche des rameaux. Il jouissait également d'une place particulière lors de la célébration des stationes, ces actions liturgiques menées par l'archevêque et le clergé urbain. Cette position de première des abbayes tréviroises se marque encore dans les listes de témoins des synodes diocésains de cette époque : le nom de l'abbé y figure après celui des archidiacres, du prévôt du chapitre cathédral et des prévôts des grands chapitres collégiaux et devance ceux des autres abbés : Die Benediktinerabtei, vol. 1, p. 269-293. Cette position prédominante doit toutefois être nuancée. Pour P. BECKER, « Die monastische Observanz in den Trierer Abteien St. Eucharius-St. Matthias und St. Maximin bis zum 15. Jahrhundert. Versuch eines Überblicks ", Kurtrierisches Jahrbuch, 7 (1967), p. 28, c'est l'abbaye bénédictine de Saint-Matthias qui jouissait du statut de primus inter pares. En 1218, le pape Honorius III chargea en effet les abbés des abbayes bénédictines de Saint-Matthias de Trèves et de SaintÈvre de Toul, ainsi que les abbés cisterciens d'Himmerod et de Wörschweiler, de réformer les abbayes bénédictines de la province ecclésiastique de Trèves. Le choix de Saint-Matthias au détriment de Saint-Maximin s'expliquerait par le fait que l'ancienne abbaye impériale n'avait pas su surmonter son passage au statut d'Eigenkloster épiscopal. Pourtant, les efforts considérables déployés par les abbés successifs de la première moitié du XIII ${ }^{\mathrm{e}}$ siècle ainsi que le soutien de l'archevêque Thierry II de Wied contribuèrent à redonner à SaintMaximin une certaine vigueur. La situation s'explique en fait davantage par le contexte politique de l'époque avec le conflit opposant l'empereur Frédéric II de Hohenstaufen à la papauté. Alors que l'archevêque de Trèves soutenait le parti impérial, l'abbé de Saint-Matthias, Jacob (1212-1257), qui était issu de la famille des ducs de Lorraine, prit soin de se maintenir à l'écart des querelles et continua à entretenir de bonnes relations avec la papauté. Ceci expliquerait dès lors l'importance toute particulière accordée par Thierry II de Wied à Saint- 
de quatre nouveaux autels au niveau de la crypte extérieure par l'archevêque Thierry II de Wied.

Entretemps, l'église, menacée par la ruine et lourdement endommagée à la suite d'un incendie, fut l'objet d'importants travaux et se fit nouvellement consacrée avec huit autels le 28 juin 1245 par l'archevêque de Cologne en présence de l'élu de Trèves, Arnold d'Isenbourg, de l'archevêque de Mayence ainsi que d'autres évêques ${ }^{21}$. Cette époque vit également la mise en place du liber aureus, un cartulaire prestigieux rassemblant les actes entérinant les droits et les privilèges de l'abbaye ${ }^{22}$. À côté de ce dernier, l'atelier de l'abbaye se lança également dans une

Maximin. Dans une contribution plus récente, Becker insistait d'ailleurs sur le fait que l'âge d'or de Saint-Matthias fut révolu dès le début du siècle : P. BECKER, «Trier. St. Eucharius - St. Matthias », dans Die Männer-und Frauenklöster der Benediktiner in Rheinland-Pfalz und Saarland, éd. F. JÜRGENSMEIER, Sainte-Odile, 1999 (Germania Benedictina, 9), p. 906-907.

${ }^{21}$ Die Benediktinerabtei, vol. 1, p. 70 et 286. Les notes de dédicace des autels de 1231 sont éditées dans les $M G H$ $S S$, vol. 15,2, p. 1269-1272. Aucun détail ne nous est parvenu concernant les reliques des huit autels consacrés en 1245. En tout cas, la reparatio chori eut encore des résonnances aux siècles ultérieurs. Dans sa Series abbatum imperialis monasterii S. Maximini, le jésuite luxembourgeois Alexander Wiltheim (1604-1684) accorda à cet événement une importance toute particulière, le faisant figurer parmi les six grands événements ayant marqué l'histoire du monastère jusqu'à son époque (cf. Bruxelles, Bibliothèque Royale, II 6835-36) : 1) La fondation de l'abbaye sur ordre de Constantin et de sa mère Hélène ; 2) Les destructions successives par les Vandales (433) et par les Huns (453) ; 3) La destruction par les Normands (883), la redécouverte miraculeuse du corps de saint Maximin et la reconstruction par l'abbé Ogon, futur évêque de Liège ; 4) La restauration à proprement parler du chœur et sa consécration en 1245 ; 5) La destruction de l'abbaye après le départ du mercenaire François de Sickingen (1522) et sa reconstruction sous l'abbé Jean III Schienen de Zell en 1533 ; 6) La destruction de l'abbaye par le feu sur ordre du marquis de Brandebourg en 1553. Texte édité dans Compte-rendu des séances de la commission royale d'Histoire, ou recueil de ses bulletins, 5 (1842), p. 7-31, voir particulièrement les p. 7 et 16. Le moine Nicolas Novillanius (1579-1618), auteur du Chronicon Imperialis Monasterii S. Maximini O. S. B. prope Treviros. Ab anno Christi CCCXXXIII. ad annum MDLXXXII., n'est pas non plus avare d'informations et fournit le détail des notes de dédicaces des autels consacrés en 1231. Le texte est édité dans J. N. VON HoNTHEIM, Prodromus Historiae Trevirensis, vol. 2, Augsbourg, 1757, p. 1019-1022. Au sujet de Novillanius, voir Die Benediktinerabtei, vol. 2, p. 1180-1182.

${ }^{22}$ Tirant leur appellation de leurs couvertures précieuses, les Libri aurei sont de prestigieux cartulaires propres aux abbayes de Prüm, d'Echternach et de Saint-Maximin de Trèves. Alors que celui de Prüm fut établi dès le début $\mathrm{du} \mathrm{XII}^{\mathrm{e}}$ siècle, le deuxième et le troisième datent tous deux du tournant du XIII ${ }^{\mathrm{e}}$ siècle. Or, ces Libri aurei d'Echternach et de Saint-Maximin ont en commun l'intervention de l'abbé Bartholomé d'Esch, qui détenait Echternach et Saint-Maximin en union personnelle depuis 1214. Du Liber aureus maximinien, détruit après la sécularisation de l'abbaye en 1807 , on ne conserve que deux copies dressées respectivement au XIV siècle Coblence, Landeshauptarchiv, Best. 211, $\mathrm{n}^{\circ} 2111$, f. $4 \mathrm{~s}-$ et au XVII ${ }^{\mathrm{e}}$ siècle sous l'abbatiat d'Alexandre Henn (1680-1698) - Trèves, Stadtbibliothek, 1632/396, p. 1-343 - ainsi qu'une plaquette d'ivoire provenant de la couverture. Cette dernière fut cependant détruite en 1945 lors d'un bombardement de Berlin. Les copies manuscrites du Liber aureus présentent l'avantage d'indiquer la foliotation d'origine du document. Compilant un cartulaire (fol. 6r-78v), un polyptyque (fol. 79r-125v) et un registre de rentes (fol. 126v-139r), l'ouvrage constitue une actualisation de documents préexistants établis à la charnière des $\mathrm{XII}^{\mathrm{e}}$ et XIII ${ }^{\mathrm{e}}$ siècles. Impossible cependant de préciser, sur la base des seules copies, le moment précis auquel le travail a abouti. Le dernier acte concernant l'abbatiat de Bartholomé remonte à 1228, bien que le noyau dur du Liber ait pu être fixé dès 1219, 1222 ou 1225. Outre son programme diplomatique, le Liber aureus de Saint-Maximin est surtout connu pour sa couverture précieuse, fabriquée entre 1220 et 1240 et attribuée à un groupe d'orfèvres actifs dans les années 1225-1250. Malheureusement détruite, on ne conserve de cette dernière qu'une vue d'artiste à l'aquarelle et une gravure de détail datant respectivement de 1731 et 1638. Si l'ensemble des bienfaiteurs royaux de l'abbaye, légendaires ou attestés, est représenté sur cette couverture, celle-ci met avant tout l'accent sur les possessions de l'abbaye puisque les noms des donations sont associés aux figures des bienfaiteurs. À l'instar du contenu du manuscrit, elle n'avait donc pas pour objectif de manifester une volonté de recouvrer le statut perdu de Reichsabtei mais plutôt d'attester de l'ancienneté de l'histoire abbatiale, d'adapter celle-ci au nouveau statut d'abbaye épiscopale et enfin de consolider et d'ancrer par l'écrit les possessions de l'institution. La mise par écrit d'un document tel que le Liber aureus a nécessairement été réalisée au sein du scriptorium de l'abbaye, seul endroit où la manipulation, la copie et la réécriture du corpus issu du chartrier a pu se concrétiser. R. NoldEN, Das goldene Buch von Prüm : Liber 
vaste campagne de production de manuscrits à laquelle se rattachent les volumes du grand légendier ${ }^{23}$. Cette entreprise, à laquelle on peut imputer pas moins de 16 manuscrits ainsi que des fragments, fut probablement chapeautée par le prieur Frédéric qui est attesté pour les années 1232 à $1240^{24}$. Son nom est référencé dans deux des volumes du grand légendier ainsi que dans un volume des gloses sur les épîtres de Paul de Pierre Lombard, aujourd'hui en mains privées ${ }^{25}$. Par ailleurs, la mémoire du prieur est également célébrée dans l'obituaire du livre du chapitre où l'on insiste notamment sur son rôle dans les activités livresques de l'institution ${ }^{26}$.

Tout en tenant compte des aléas de la conservation, le nombre très limité de manuscrits hagiographiques provenant de Saint-Maximin antérieurs au grand légendier permet de supposer que c'est probablement sur la base d'une autre collection que la plupart des textes des volumes du grand légendier furent copiés ${ }^{27}$. Or, la comparaison de son sanctoral avec celui de plusieurs autres reliquats de légendiers provenant de l'abbaye voisine de Saint-Matthias a permis de mettre en évidence un tronc commun qui pose la question de la diffusion d'une collection dans la région de Trèves vers le tournant des XII $-\mathrm{XIII}^{\mathrm{e}}$ siècles $^{28}$.

Quant au sanctoral du légendier, la confrontation des volumes de la collection avec une série de documents, essentiellement des calendriers, montre que les textes dédiés aux saints les plus importants de l'abbaye, notamment ceux dont elle possédait des reliques, figurent bel et

aureus Prumiensis (StB Trier, Hs 1709). Ein Kopiar mit Urkundenabschriften des 8. bis 12. Jahrhunderts, Trèves, 2013 (Kostbarkeiten der Stadtbibliothek Trier, 4) ; M. MARGUE, "Libertas ecclesiae. Réformes monastiques et réécriture de l'histoire dans l'espace lotharingien ( $\mathrm{X}^{\mathrm{e}}-\mathrm{XII}{ }^{\mathrm{e}} \mathrm{s}$.). Le cas de l'abbaye d'Echternach », dans Écrire son histoire. Les communautés régulières face à leur passé. Actes du $5^{e}$ colloque international du C.E.R.C.O.R. ; SaintÉtienne, 6-8 novembre 2002, éd. N. BouTER, Saint-Étienne, 2006 (Travaux et recherches, 18), p. 107123 ; C. SAUER, Fundatio und Memoria. Stifter und Klostergründer im Bild (1100 bis 1350), Göttingen, 1993 (Veröffentlichungen des Max-Planck-Instituts für Geschichte, 109), p. 215-216 et 246-279; H. W. KUHN, « Das politische Programm des Liber aureus von St. Maximin (Trier). Untersuchungen über Chartular und Prachteinband aus dem 13. Jahrhundert », Jahrbuch für westdeutsche Landesgeschichte, 4 (1978), p. 81-128.

${ }^{23}$ Cf. B. Dubuisson, « Bibliothéconomie ».

24 Die Benediktinerabtei, vol. 2, p. 1153-1154; G. KENTENICH, « Das Trierer Kunsthandwerk in seiner geschichtlichen Entwicklung », Trierische Heimatblätter, 1 (1922), p. 62-64 et 177-183.

${ }_{25}$ «Hunc librum comparavit bone memorie prior Fridericus ». Cf. Paris, BNF, lat. 9741, p. 452 ; lat. 9742 , p. 321 ; K. VON RózYCKI, « Die Freiherrlich v. Cramer-Klettsche Schloßbibliothek in Hohenaschau », Zeitschrift für Bücherfreunde, Neue Serie, 7 (1915/16), p. 115.

${ }^{26}$ Trèves, Stadtbibliothek, 1634/394, fol. 139v-151v (ca. 1260), 20 mai (fol. 144r) : « Fridericus prior nostrae congregationis qui hanc ecclesiam in aedificis, libris et ornamentis multum emendavit». Cette mention nécrologique relative à un membre de la communauté est la seule qui ait été intégralement transcrite à l'encre rouge à côté du nom des grands bienfaiteurs princiers et archiépiscopaux. Les noms des abbés Bartholomé d'Esch (14/11) et Henri III de Bruch (28/02) ont quant à eux été soulignés d'un trait rouge. Dans un nécrologe présent dans le même manuscrit (fol. 93r-139r, ca. 1180), une main postérieure a ajouté à l'encre rouge la mention du prieur Frédéric, mais au 21 mai (Fridericus prior, sacerdos et monachus nostrae congregationis). À propos de ce nécrologe, cf. D. GEUENICH, «Eine unveröffentlichte Verbrüderungsliste des 12. Jahrhunderts aus St. Maximin/Trier », Rheinische Vierteljahrsblätter, 41 (1977), p. 180-195 ; F. RoBERG, Das älteste 'Necrolog' des Klosters St. Maximin vor Trier, Hanovre, 2008 (Monumenta Germaniae Historica; Libri memoriales et necrologia ; Nova Series, 8), p. 58.

${ }^{27}$ Cf. B. Dubuisson, « Bibliothéconomie »

${ }^{28}$ Cf. n. 13 et 19 . 
bien au sein de la collection. Il faut préciser que c'est essentiellement grâce à deux documents figurant au sein du livre du chapitre, soit le martyrologe ainsi qu'une liste très fournie de Vies et de passions de saints organisée sous la forme d'un calendrier ${ }^{29}$, que je suis parvenu à rétablir les dates de célébration d'un certain nombre de saints dont la présence au sein de tel ou tel volume pouvait paraître incongrue, d'autant plus que tous ne faisaient pas partie du sanctoral maximinien traditionnel.

Avant de passer au point suivant, il faut relever que, dans le grand légendier, le classement de la matière hagiographique suivant le déroulement de l'année liturgique n'est pas strictement respecté ${ }^{30}$. En effet, chaque volume peut être scindé en blocs de légendes induisant des ruptures du circulus anni qui traduisent très certainement un travail par groupes de copistes $^{31}$. Par ailleurs, il convient également de souligner que le légendier n'a pas été intégralement terminé au XIII ${ }^{\mathrm{e}}$ siècle. En effet, une série d'initiales ainsi que les titres rubriqués de plusieurs textes, dont certains dédiés à des saints de première importance dans le sanctoral de l'abbaye, y compris des saints patrons, n'avait tout simplement pas été apposés.

Dès lors, le caractère non systématique du classement per circulum anni, l'absence de la date de la fête des saints dans les intitulés rubriqués des légendes, voire l'absence pure et simple de ces titres devait faire du grand légendier un ensemble de volumes assez peu pratique pour les lectures quotidiennes aux offices ou au réfectoire. Ce n'est d'ailleurs peut-être pas un

\footnotetext{
${ }^{29}$ Trèves, Stadtbibliothek, 1634/394, fol. 1-6 (calendrier hagiographique), fol. 15v-56r (martyrologe).

${ }^{30} \mathrm{Il}$ s'agit là d'un trait propre aux grandes collections : la copie des textes se faisait parfois au compte-gouttes, en fonction de l'arrivage de modèles qui pouvait s'étaler dans le temps. Voir à ce sujet le témoignage du compilateur d'un légendier du XII ${ }^{\mathrm{e}}$ siècle de l'abbaye de Fulda: B. VOGEL, «Hagiographische Handschriften im 12. Jahrhundert », dans Europa an der Wende vom 11. zum 12. Jahrhundert. Beiträge zu Ehren von Werner Goez, éd. K. HERBERS, Stuttgart, 2001, p. 211.

${ }^{31}$ L'uniformité du grand légendier se marque au niveau de la mise en texte des volumes puisqu'une même écriture praegothica a servi de modèle de référence à l'ensemble des copistes. Plusieurs collaborateurs ont œuvré à la transcription des textes et se sont fréquemment relayés. Toutefois, l'homogénéité qui se dégage de leur travail rend toute identification des mains extrêmement délicate. L'étudier impliquerait par ailleurs de se pencher plus en avant sur les autres manuscrits liés à la même campagne de production. Sur la base de la structure codicologique que j'ai dégagée pour chaque volume, il est toutefois possible de repérer des «blocs» de légendes couvrant chacun un nombre aléatoire de jours. C'est l'agencement de ces ensembles, les uns à la suite des autres, qui constitue en quelque sorte la colonne vertébrale de chaque volume. Chaque ensemble commence et se termine par une légende, de sorte qu'aucune œuvre ne chevauche deux cahiers à l'endroit de la jonction des blocs. Souvent, le saut d'un bloc à un autre se traduit par un changement de main, parfois aussi par un changement du procédé de réglure (pointe sèche ou pointe traçante). Les copistes semblent donc avoir agi en groupes de travail chargés de transcrire un certain nombre d'œuvres. Le produit de leur labeur respectif, s'articulant en une série de cahiers, fut ensuite adjoint à celui des autres pour former finalement les différents volumes du légendier. À propos de la collaboration entre copistes, voir les articles regroupés à ce sujet dans La collaboration dans la production de l'écrit médiéval. Actes du XIII ${ }^{e}$ colloque du Comité International de Paléographie Latine, éd. H. SPILling, Paris, 2003 (Matériaux pour l'histoire, 4). La subdivision du travail par groupes de scribes a également été mise en évidence par M. MANIACI concernant la production de «bibles atlantiques » (i.e. bibles de grand format, produites à partir du $\mathrm{XI}^{\mathrm{e}}$ siècle). La mise par écrit des différents livres était confiée à différentes équipes, après quoi le travail de chacun était assemblé, ce qui donnait parfois lieu à certaines incohérences : parmi d'autres travaux, voir : «L'officina delle Bibbie atlantiche: artigiani, scribi, miniatori », dans Come nasce un manoscritto miniato. Scriptoria, tecniche, modelli e materiali, éd. F. Flores D’ARCAIS, F. Crivello, Modène, 2010, p. 197-212.
} 
hasard si les seules annotations évoquant clairement un tel usage remontent à la toute fin du Moyen Âge, après que le grand légendier ait subit un certain nombre d'interventions destinées à en améliorer la consultation ${ }^{32}$. Ces modifications s'échelonnèrent jusqu'au début du XVI ${ }^{\mathrm{e}}$ siècle et leur compréhension nécessite tout d'abord une remise en contexte plus large.

\section{Les entreprises bibliothéconomiques des $\mathrm{XIV}^{\mathrm{e}}-\mathrm{XVI}^{\mathrm{e}}$ siècles}

À Saint-Maximin de Trèves, le regain d'activité de la première moitié du XIII ${ }^{\mathrm{e}}$ siècle fut suivi d'une période moins faste dès la seconde moitié du XIV siècle. Alors que la communauté regroupait 35 à 45 moines au XIII ${ }^{\mathrm{e}}$ siècle, ce nombre chuta à neuf en 1373 puis à six ou cinq en 1434. Par ailleurs, certaines parties des bâtiments partirent en fumée en 1325 tandis qu'une série de conflits opposèrent l'abbaye à l'archevêque et aux bourgeois de Trèves, les seconds allant jusqu'à détruire l'abbaye en 1433/1434 avec l'assentiment du premier. Enfin, au niveau économique, la situation de l'institution était devenue déplorable, du fait notamment d'abbés dépensiers, si bien que dans les années 1440, l'abbaye était criblée de dettes et menaçait faillite $^{33}$. Face à cette dégradation de la vie monastique et de la situation matérielle de l'abbaye, plusieurs tentatives de redressement furent initiées ${ }^{34}$.

Dans la seconde moitié du XIV siècle, l'abbé Rorich d'Eppelborn (1369-1411), lança un projet de restauration, marqué notamment par le renouvellement de la liturgie et une tentative d'assainissement des finances ${ }^{35}$. Bien que l'on connaisse assez mal les tenants et aboutissants de ses actions, on conserve de cette époque un inventaire daté du 13 mai 1393 recensant des livres, seul autre document fournissant un aperçu du contenu de la bibliothèque à côté du catalogue du XII ${ }^{\mathrm{e}}$ siècle qui détaillait celui de l'armarium ${ }^{36}$.

\footnotetext{
${ }^{32}$ On relève deux annotations marginales remontant au tournant des $\mathrm{XV}^{\mathrm{e}}-\mathrm{XVI}^{\mathrm{e}}$ siècles invitant à poursuivre la lecture au réfectoire et au chœur. Paris, Bibliothèque nationale de France, lat. 9742, p. 71 : «Hoc continuatur in refectorio »; Trèves, Bibliothek des Bischöflichen Priesterseminars, 36, fol. 138r : "Hic continuatur lectiones in choro $\gg$.

${ }^{33}$ Les problèmes de recrutement, les conflits et faits de guerre ainsi que les problèmes financiers sont en effet les maux qui frappèrent de nombreux établissements monastiques à cette époque. P.-J. DE GRIECK, De benedictijnse geschiedschrijving, p. 92-98.

34 Pour un aperçu général des tentatives de restauration de la vie monastique à Saint-Maximin, cf. Die Benediktinerabtei, vol. 1, p. 290-293 (XIV siècle) et p. 294-316 (XV siècle).

${ }^{35} \mathrm{Au}$ sujet de l'abbé Rorich, cf. Die Benediktinerabtei, vol. 2, p. 1076-1079. Outre l'inventaire dont il sera ici question, il fit également rédiger un ordinaire pour réorganiser la liturgie (Trèves, Stadtbibliothek, 1635/48, fol. 60r-87v) : P. BECKER, « Die monastische Observanz », p. 31.

${ }^{36}$ Cet inventaire est transcrit à la toute fin d'un manuscrit aujourd'hui conservé aux Landeshauptarchiv à Coblence (Best. 211, n 2111, p. 168-170), à la suite d'une copie du Liber aureus. Il est édité, de même que le catalogue du $\mathrm{XII}^{\mathrm{e}}$ siècle, dans M. KEUFFER, « Bücherei und Bücherwesen von S. Maximin im Mittelalter », Jahresbericht der Gesellschaft für nützliche Forschungen zu Trier von 1894 bis 1899, (1899), p. 48-94 (p. 54-58). Voir également I. KNOBLICH, Die Bibliothek des Klosters St. Maximin bei Trier bis zum 12. Jahrhundert, Trèves, 1996.
} 
Revêtant un caractère officiel, ce document fut rédigé sur ordre de l'abbé par le prêtre de Tylo, au sujet duquel on ne sait rien, en présence du prieur Engelbert de Blankenheim et du moine Nicolas de Redeling le 13 mai 1393, jour de la Saint-Servais ${ }^{37}$. Qualifié à tort de « catalogue $»^{38}$, cet inventaire témoigne d'une profonde méconnaissance des livres détenus par l'institution, hormis ceux utilisés quotidiennement au chœur pour la liturgie ${ }^{39}$, et ainsi d'une volonté de réagir contre le profond laisser-aller dans la gestion de la bibliothèque ${ }^{40}$. En tête des ouvrages listés, on retrouve les neuf volumes du grand légendier ${ }^{41}$.

L'établissement de ce document serait donc l'une des premières tentatives de réorganisation du fonds de livres ${ }^{42}$. L'entreprise lancée par l'abbé Rorich ne fut toutefois que

${ }^{37} \mathrm{Au}$ sujet d'Engelbert de Blankenheim et de Nicolas de Redeling, cf. Die Benediktinerabtei, vol. 2, p. 1157 et 1235.

38 «Le catalogue se distingue du simple inventaire par la présence d'une classification (alphabétique par noms d'auteurs et titres d'anonymes, alphabétique par sujet, systématique par matières, ou autre) » : A. DEROLEZ, Les catalogues de bibliothèques, Turnhout, 1979 (Typologie des sources du Moyen Âge occidental, fasc. 31), p. 15 ; cet aspect est en l'occurrence absent du document de 1393.

39 «Notandum, quod omnes libri, quibus utimur in choro, non sunt registrati; sed sola volumina librorum in byblioteca scripta sunt $»$. Il existait peut-être un autre catalogue recensant également les manuscrits du chœur. En effet, l'intitulé du document, "Primo libri collacionum », laisse entendre que d'autres inventaires de la sorte existaient. Nous n'en avons malheureusement aucune trace. Le terme de «collatio » renverrait ici au sens de « réunion » ou d'« assemblage », en ce sens qu'on aurait rassemblé tous les livres de la bibliothèque (peut-être dans des coffres ou dans des armoires) pour en dresser l'inventaire. On aurait également pu interpréter le terme comme renvoyant à la collatio monastique, ce qui aurait alors fait de cet inventaire un répertoire des livres qui pouvaient être lus au chapitre. Toutefois, la différence clairement établie entre les livres de la bibliothèque et les livres utilisés au chœur, le caractère officiel du recensement ainsi que le nombre très important d'ouvrages référencés de nature très diverse rend ce raisonnement peu probable. Pour comparer, on pourra consulter d'autres listes de lecture pour la collatio : D. NeBBiAi-DAlla GuARDA, « Les listes médiévales de lectures monastiques. Contribution à la connaissance des anciennes bibliothèques bénédictines », Revue bénédictine, 96 (1986), p. 271326. Cf. également Fr. Dolbeau, «À propos des lectures de table. Présentation de trois calendriers cisterciens renvoyant à des légendiers ", Les cisterciens et la transmission des textes (XII $-X V I I I^{e}$ siècles), éd. Th. FALMAGNE, D. Stutzmann, A.-M. Turcan-Verkerk, Turnhout, 2018 (Bibliothèque d'histoire culturelle du Moyen Âge, 18), p. 402-403 et 404-405.

${ }^{40}$ Les manuscrits sont listés en groupes, pêle-mêle, les uns à la suite des autres sans que transparaisse de véritable logique de classement. À la fin de chaque regroupement, on a indiqué la somme des volumes en "pièces entières » (summa voluminum pecie integre). Au total, 159 volumes ou pièces sont ainsi recensés (summa summarum voluminum : CLIX pecie integre), abstraction faite d'une série de livres jugés de moindre valeur (Item multi alii libri modici valoris). L'utilisation du terme « pecia» renvoie à une notion employée dans le monde universitaire où les œuvres étaient reproduites à la chaine par le système des «peciae», lui-même inspiré de la subdivision du travail des moines copistes au sein des scriptoria monastiques : H. V. SCHOONER, «La production du livre par la pecia», dans La production du livre universitaire au Moyen Age. Exemplar et pecia, éd. L. J. BATAILLON, G. GuYot, R. H. Rouse, Paris, 1991, p. 17-37. Dans le contexte de l'inventaire, le terme de «pecia » ne renvoie cependant pas à ce système mais est employé comme synonyme de « volumen », puisque l'addition des titres listés correspond bien aux sommes indiquées à la fin de chaque regroupement ainsi qu'au total final. Il convient de noter qu'à la suite de chaque élément listé, on donne des précisions d'ordre matériel sur le volume en question («in uno/magno/parvo/antiquo/... volumine»). À une seule occasion, le même volume est cité à deux reprises («in eodem volumine ») mais n'est finalement comptabilisé qu'une seul fois. On l'aura compris, ici, le terme de "pecia» doit donc être compris dans son acception «d'unité de compte»: L. J. BATAILlON, «Exemplar, pecia, quaternus", dans Vocabulaire du livre et de l'écriture au moyen âge. Actes de la table ronde. Paris 24-26 septembre 1987, éd. O. WeIJERS, Turnhout, 1989 (Études sur le vocabulaire intellectuel du Moyen Âge, 2), p. 210211.

${ }^{41}$ Cf. B. DuBuisson, « Bibliothéconomie », en part. les tableaux annexes.

${ }^{42}$ Le contenu de l'ancien armarium, tel qu'il transparaît dans le catalogue du XII ${ }^{\mathrm{e}}$ siècle et auquel il faut ajouter les manuscrits produits avant 1393, avait sans doute été dispersé à travers les bâtiments claustraux suivant les 
la première étape d'un long travail qui allait connaître ultérieurement des développements plus importants.

En 1436, deux ans après avoir été détruite à la suite d'une querelle avec l'élu de Trèves, Ulrich de Manderscheid, l'abbaye de Saint-Maximin introduisit en l'adaptant le coutumier de Jean Rode (†1439), abbé et réformateur de l'abbaye voisine de Saint-Matthias de Trèves ${ }^{43}$. Dans les années qui suivirent, l'abbaye retrouva un certain dynamisme mais surtout une nouvelle attractivité puisqu'elle fut ouverte aux membres de la bourgeoisie et non plus exclusivement réservée à la noblesse ${ }^{44}$. De huit moines en 1449, la communauté s'agrandit progressivement jusqu'à réunir 27 membres en 1516. Ce regain d'activité se marqua également dans la gestion de la collection de livres.

C'est sans doute sous l'abbatiat d'Antoine II de Drüblein (1452-1482), ancien prieur de Saint-Matthias, promu à la tête de Saint-Maximin par le célèbre penseur Nicolas de Cues et le pape Nicolas $\mathrm{V}$, que ces initiatives importantes furent lancées ${ }^{45}$. Elles se perpétuèrent sous l'abbatiat d'Othon IV d'Elten (1483-1502) qui envoya en 1496 douze de ses moines réformer l'abbaye d'Echternach où une entreprise similaire fut lancée ${ }^{46}$.

\footnotetext{
besoins des moines. Outre cet aléa de la conservation, il faut également tenir compte des « accidents » naturels ou causés par l'homme (vols, humidité, feu, parasites et rongeurs). Ce n'est sans doute pas un hasard si le mot «byblioteca » est désormais expressément mentionné comme lieu de conservation des manuscrits. On voit en effet apparaître, à partir du XIII ${ }^{\mathrm{e}}$ siècle, les premières véritables bibliothèques monastiques. Celles-ci remplacèrent progressivement les armaria, ces petits locaux, dépôts ou armoires qui avaient prévalu jusqu'au XII ${ }^{\mathrm{e}}$ siècle. La première mention d'une bibliothèque pour Saint-Maximin, à l'extrême fin du XIV ${ }^{\mathrm{e}}$ siècle, doit dès lors être mise en lien avec la multiplication des bibliothèques monastiques aux derniers siècles du Moyen Âge. X. HERMAND, "Comment les communautés religieuses géraient-elles leur bibliothèque (Pays-Bas méridionaux, $\mathrm{XIV}^{\mathrm{e}}-\mathrm{XV}^{\mathrm{e}}$ siècles) ? ", dans Décrire, inventorier, enregistrer entre Seine et Rhin au Moyen Age. Formes, fonctions et usages des écrits de gestion. Actes du colloque international organisé à l'université de Namur (FUNDP) les 8 et 9 mai 2008, éd. X. Hermand, J.-F. Nieus, É. RenARd, Paris, 2012 (Mémoires et documents de l’École des Chartes, 92), p. 355-361.

${ }_{43}$ Die Benediktinerabtei, vol. 1, p. 294-298. Le coutumier est édité dans Consuetudines et observantiae monasteriorum Sancti Mathiae et Sancti Maximini Treverensium ab Iohanne Rode abbate conscriptae, éd. P. BeCKer, Siegburg, 1968 (Corpus Consuetudinum Monasticarum, 5). Pour son commentaire, cf. Id., Das monastische Reformprogramm des Johannes Rode, Abtes von St. Matthias in Trier, Münster, 1970 (Beiträge zur Geschichte des alten Mönchtums und des Benediktinerordens, 30). Sur la réforme de Rode, on se réfèrera aux nombreuses autres publications de P. Becker.

${ }^{44}$ Id., « Die ständische Zusammensetzung der Abteien St. Matthias und St. Maximin in Trier zu Beginn der Reform des Abtes Johannes Rode (†1439)», Archiv für mittelrheinische Kirchengeschichte, 18 (1966), p. 313-320.

${ }^{45}$ Au sujet de cet abbé cf. Die Benediktinerabtei, vol. 2, p. 1087-1089.

${ }^{46} \mathrm{Ibid}$., p. 1090-1091 ainsi que vol. 1, p. 153. Les activités menées par les moines de Saint-Maximin à Echternach permirent de renouveler le lien étroit qui unissait les deux abbayes. En 1511, l'abbé Thomas de Huisdem offrit un rituel à sa consœur luxembourgeoise (Luxembourg, Bibliothèque nationale du Luxembourg, 136). P. BECKER, Das monastische Reformprogramm, p. 179 ; Id., « Bemühungen um eine geistliche Erneurerung der Abtei Echternach. Eine Visitation im Jahre 1443 ", dans Willibrord: Apostel der Niederlande, Gründer der Abtei Echternach, éd. G. Kiesel, J. Schroeder, Luxembourg, 1989, p. 294. Voir également R. Nolden, «Zu den ContinetEinträgen in den Echternacher Handschriften in Trier », dans Analecta Epternacensia. Beiträge zur Bibliothekgsgeschichte der Abtei Echternach, éd. L. DeITZ, R. NOLDEN, Luxembourg, 2000, p. 71-82.
} 
Influencés par les travaux menés parallèlement à Saint-Matthias ${ }^{47}$, les moines de SaintMaximin dotèrent la majorité de leurs livres de cotes, d'indications de contenu et d'ex-libris, systématiquement apposés par deux ou trois mains récurrentes ${ }^{48}$. L'introduction de ces procédés témoigne non seulement d'une volonté de structuration du fonds de livres afin de mieux guider le lecteur désireux de trouver un ouvrage particulier mais aussi d'un souci de conservation accru de la part des moines qui allait de pair avec la naissance de véritables bibliothèques monastiques ${ }^{49}$.

À l'image de ce que l'on constate à Saint-Matthias et à Echternach, les cotes attribuées aux livres de Saint-Maximin renvoyaient à un classement thématique et étaient formées d'une lettre suivie d'un numéro d'ordre. Ainsi, le grand légendier et une majorité d'autres manuscrits hagiographiques furent classés sous la lettre $« \mathrm{~N} »^{50}$. Ces cotes ont généralement été inscrites sur le premier recto des manuscrits, incunables ou livres imprimés mais peuvent également avoir été répétées à d'autres endroits des volumes. Elles figuraient par ailleurs sur le dos des

47 À Saint-Matthias, la réforme introduite par Jean Rode se marqua par un soin particulier apporté à la réorganisation et au renouvellement de la bibliothèque. Elle fut remodelée pour répondre à une utilisation quotidienne portant sur l'étude, les lectures spirituelles et la pastorale. Cependant, pareille initiative entraina également la perte et le démantèlement de nombreux manuscrits jugés vétustes et devenus peu lisibles : P. BECKER, Das monastische Reformprogramm, p. 116 ; Id., «Die Trierer kirchlichen Bibliotheken. Geistesgeschichtliche Aspekte aus verschiedenen Jahrhunderten », dans Armaria Trevirensia. Beiträge zur Trierer Bibliotheksgeschichte, éd. G. Franz, Wiesbaden, 1985 (Bibliotheca Trevirensis, 1) p. 8-10.

${ }^{48}$ Die Benediktinerabtei, vol. 1, p. 152-153.

${ }^{49}$ X. HeRMAND, « Comment les communautés », p. 389-390.

${ }^{50} \mathrm{~L}$ 'interprétation la plus simple et la plus usuelle consiste à faire correspondre chaque lettre de l'alphabet à un meuble ou partie de meuble tandis que le numéro serait relatif à la position du livre parmi les volumes déposés à cet endroit: ibid., p. 391. Toutefois, Th. FALmagne a souligné que le système de cotation de l'abbaye d'Echternach, importé par les douze moines réformateurs de Saint-Maximin en 1496 puis perpétué et systématisé par le bibliothécaire Willibrord Schramm de 1526 à 1541, se basait sur un classement des ouvrages par genre littéraire (ex. : «I» = hagiographie et histoire). Die Echternacher Handschriften bis zum Jahr 1628 in den Beständen der Bibliothèque nationale Luxembourg sowie der Archives diocésaines de Luxembourg, der Archives nationales, der Section historique de l'Institut grand-ducal und des Grand Séminaire de Luxembourg, vol. 1, Wiesbaden, 2009, p. 123-127. La même logique prévalait à l'abbaye de Saint-Matthias pour laquelle on conserve la copie d'un catalogue reprenant ces cotes (Trèves, Stadtbibliothek, 2229/1751). Il existait en effet au bas Moyen Âge une tendance à privilégier un classement des ouvrages par matières, influencé par les classifications scolastiques des sciences : A. Derolez, Les catalogues, p. 29 et 32-33. Bien entendu, ces logiques de classement thématique n'étaient pas étrangères à des systèmes rangement par meuble ou partie de meuble et pouvaient leur être complémentaires. Pour revenir à Saint-Maximin et au classement de la matière hagiographique, les huit volumes subsistant du grand légendier portent les cotes « N 2-9». J'ai relevé toute une série d'autres manuscrits hagiographiques portant une cote débutant par la lettre « $\mathrm{N} »$ : Londres, Society of Antiquaries, 279 (légendier abrégé de Jean de Mailly, «N $11 »)$; Paris, Bibliothèque nationale de France, lat.9742, p. 323-492 (Vie et miracles de Bernard de Clairvaux suivi du Liber prognosticorum futuri saeculi de Julien de Tolède, « N $14 »)$; Vienne, Österreichische Nationalbibliothek, cod. 490, 541 et 1052 (libelli contenant les Vies des saints Guibert, Malo, Germain et Conrad, « N $16 »)$; Bruxelles, Bibliothèque Royale, II 2611 (Vie de saint Remacle, «N $20 »)$; Berlin, Staatsbibliothek Preußischer Kulturbesitz, theol. lat. fol. 729 (Legendae patronorum monasterii S. Maximini Trevirensis, « N $22 »)$. 
reliures des ouvrages, bien que peu de témoins subsistent du fait des campagnes de reliure successives ${ }^{51}$.

Les travaux entrepris ne se limitèrent toutefois pas à la seule apposition de cotes mais prirent également la forme de restructurations ou de réassemblages de manuscrits, généralement dans le cadre de campagne de mise en reliure. J'y reviendrai en ce qui concerne les volumes du grand légendier. Les interventions sur les livres de l'institution se poursuivirent encore dans le premier quart du XVI ${ }^{\mathrm{e}}$ siècle jusqu'au sac de l'abbaye en 1522 par les bourgeois de la ville ${ }^{52}$. Cette époque fut en outre imprégnée par l'humanisme "monastique» (monastischer Humanismus ou Klosterhumanismus) ${ }^{53}$, par l'acquisition d'incunables puis de livres imprimés ainsi que par la production de quelques manuscrits dont la très notable Bible géante (Riesenbibel) en trois volumes commencée sous l'abbé Thomas de Huisdem (1502-1514) par le moine Vincent de Cochem et achevée par Jacob Gladbach en 1526/2754. Par son parchemin de grande qualité ${ }^{55}$, sa calligraphie soignée et ses riches enluminures, cette dernière atteste du maintien d'une production manuscrite exceptionnelle et maîtrisée à une époque où le papier et les livres imprimés prenaient le dessus ${ }^{56}$.

À l'instar de ce que l'on observe pour d'autres monastères touchés par les mouvements de l'observance ${ }^{57}$, la « réforme » inspirée de Rode s'est donc traduite sur le long terme par un

\footnotetext{
${ }^{51}$ Deux incunables de la Stadtbibliothek de Trèves - inc. 1766 («R $\left.29 »\right)$ et 1777 (« I 21/22 ») - conservent leur reliure d'origine sur laquelle figure encore la cote. Cette dernière fut transcrite à l'encre noire sur le dos des volumes, de manière à pouvoir être lue lorsque ceux-ci étaient couchés sur le plat du dessous.

${ }^{52} \mathrm{~L}$ 'abbaye fut attaquée par les bourgeois de la ville suite au départ du mercenaire François de Sickingen qui y avait établi ses quartiers lors du siège de la ville. Saint-Maximin, rappelons-le, se situait en effet extra muros. Les moines, confinés dans leur refuge urbain, ne purent que constater les destructions : cf. Die Benediktinerabtei, vol. 1, p. 318.

${ }^{53} \mathrm{La}$ terminologie pose problème dans la mesure où elle induit l'existence d'un courant parallèle, cloisonné car développé à l'ombre du cloître. Or, le courant humaniste pouvait se manifester sous différentes formes au sein des abbayes : la copie ou la rédaction d'œuvres certes, mais aussi les contacts entretenus par certains moines avec les milieux humanistes, que ce soit à travers la correspondance, les études universitaires ou la participation active à des cercles érudits : H. MÜLLER, Habit und Habitus: Mönche und Humanisten im Dialog, Tübingen, 2006 (Spätmittelalter und Reformation. Neue Reihe, 32), notamment la conclusion. Voir également P.-J. DE GRIECK, De benedictijnse geschiedschrijving, p. 509.

${ }^{54}$ Die Benediktinerabtei, vol. 2, p. 1091-1093 (Thomas de Huisdem), p. 1093-1095 (Vincent de Cochem) et p. 1230 (Jacob Gladbach). Aujourd'hui en mains privées, la Bible géante a bénéficié d'une étude détaillée de la part de F. Bezner, Von der Liturgie zur Geschichte. Die Riesenbibel von St. Maximin und die Historia Excidii Sancti Maximini, Passau, 2011 (Illuminationen, 15).

${ }^{55}$ Près d'un millier de peaux de parchemin furent achetées par les moines, notamment à Aix-la-Chapelle, comme en témoignent les comptes de l'abbaye pour les deux premières décennies des années 1500 (Trèves, Stadtbibliothek, 1626/401, p. 805-1232).

${ }^{56}$ F. BEZnER, Von der Liturgie, p. 21-23, y a vu la manifestation d'une forme de « résistance à Gutenberg et à la congrégation de Bursfeld», propos nuancés dans les Germania Sacra (Die Benediktinerabtei, vol. 1, p. 164, n. 113) : parmi les abbayes effectivement membres de la congrégation de Bursfeld, on produisait encore des manuscrits « à l'ancienne » (ex. : à Laach ou à Saint-Matthias) tandis que Saint-Maximin posséda relativement tôt un exemplaire de la Bible de Gutenberg.

${ }^{57}$ Voir par exemple X. HERMAND, « Scriptoria et bibliothèques dans les monastères cisterciens réformés des PaysBas méridionaux au $\mathrm{XV}^{\mathrm{e}}$ siècle », dans Les cisterciens et la transmission des textes (XII $-X V I I I^{e}$ siècles), éd.
} 
nouvel essor des pratiques de l'écrit à Saint-Maximin de Trèves ${ }^{58}$. Or, parmi les manuscrits produits dans la seconde moitié $\mathrm{du} \mathrm{XV} \mathrm{V}^{\mathrm{e}}$ au début du XVI $\mathrm{XIècle}^{\mathrm{e}}$ une certaine place fut accordée à l'hagiographie.

En 1461, sous l'abbatiat d'Antoine II de Drüblein (1452-1482) ${ }^{59}$, le moine Louis de Ratingen rédigea intégralement un volume de Vies des Pères, agrémenté d'une série d'autres dossiers de saints ainsi que de textes non hagiographiques (Bruxelles, Société des Bollandistes, 27) ${ }^{60}$. Comme le soulignait déjà Guy Philippart, ce manuscrit de haute facture s'avère particulièrement intéressant de par une annotation invitant le lecteur à se reporter à un « passionnaire » pour toute une série de textes ${ }^{61}$. Or, il s'agit bel et bien du grand légendier dont il est ici question puisque l'ensemble des textes indiqués s'y retrouvent et que Louis de Ratingen a personnellement pris soin d'y écrire les titres manquants de certaines de ces œuvres ${ }^{62}$. Ce renvoi explicite nous rappelle qu'il ne faut pas considérer une collection hagiographique comme un ensemble clos mais qu'il pouvait bel et bien exister une certaine complémentarité entre les différents manuscrits hagiographiques d'une institution.

D. Stutzmann, A.-M. Turcan-Verkerk, Turnhout, 2018 (Bibliothèque d'histoire culturelle du Moyen Âge, 18), p. 79-126.

${ }^{58}$ Id., « Comment les communautés », p. 357. Un phénomène identique s'est par exemple manifesté à l'abbaye de Saint-Laurent de Liège dès la première moitié $\mathrm{du} \mathrm{XV}^{\mathrm{e}}$ siècle. Une étude plus approfondie des productions et acquisitions de Saint-Maximin à la fin du Moyen Âge et au début des Temps modernes fournirait à n'en pas douter maintes informations sur l'organisation des pratiques de l'écrit à cette époque : É. TERLINDEN, «Réformes, scriptoria et bibliothèques au bas Moyen Âge : le cas de Saint-Laurent de Liège ", dans Lecteurs, lectures et groupes sociaux au Moyen Âge. Actes de la journée d'étude organisée par le Centre de recherche "Pratiques médiévales de l'écrit » (PraME) de l'Université de Namur et le Département des Manuscrits de la Bibliothèque royale de Belgique, Bruxelles, 18 mars 2010, éd. X. Hermand, É. RenARD, C. VAN HoorebeECK, Turnhout, 2014 (Texte, Codex et Contexte, 17), p. 79-121. Pour le monde germanique, on pourra également se référer aux travaux de W. Williams-Krapp qui s'est surtout intéressé aux écrits en langue allemande.

${ }^{59}$ Cf. n. 45.

${ }^{60} \mathrm{Fol} .3 \mathrm{r}$ : « Anno Domini 1461, sub venerabili patre et domino Anthonio abbate, scriptus est liber iste per fratrem Lodwicum Rathingen professum, orate pro eo ». Die Benediktinerabtei, vol. 2, p. 1319. Si le nom de Louis de Ratingen n'est attesté pour Saint-Maximin que dans ce manuscrit, il se trouve que le même individu fut ensuite moine à Saint-Matthias de Trèves (ca. 1470-1498) où il exerça la charge de custos sive thesaurarius. En 1489, il rédigea un bréviaire en deux parties pour l'infirmerie de cette abbaye (Trèves, Stadtbibliothek, 380/1049 [pars hiemalis] et 433/1928 [pars aestivalis]) : P. BECKER, Die Benediktinerabtei St. Eucharius-St. Matthias vor Trier, Berlin/New York, 1996 (Germania Sacra ; Neue Folge, 34 ; Die Bistümer der Kirchenprovinz Trier, 8), p. 710. Il décéda le 19 septembre 1498 et son nom fut inscrit dans le nécrologe du livre du chapitre (Trèves, Bibliothek des Bischöflichen Priesterseminars, 63, fol. 162r). En ce qui concerne le contenu du manuscrit de Saint-Maximin : Vitae Patrum, BHL 6524-6529, fol. 3v-29r (= livre 1), fol 29r-74r (=livre 2), fol. 75v-137v (=livre 3), fol. 143r177r (livre 4). Entre ces quatre livres sont intercalées l'une ou l'autre Vie : Vita sancti Frontini, BHL 3189, 3192, fol. 74r-75v ; Vita sancte Marine, BHL 5528, fol. 137v-139r ; Vita sancti Pachomii, BHL 6412, fol. 139r-143r ; Vita sancte Abrahe, BHL 12, fol. 177r-185r. S'ensuit une série d'autres œuvres (lettres, sermons, homélies...) ne relevant pas du genre hagiographique. Ce recueil assez dense mériterait d'être étudié pour lui-même.

${ }^{61}$ G. Philippart, Les légendiers latins, p. 55. Fol. 2v : «Vita Pauli primi heremite, Vita sancti Anthonii, Vita sancti Hilarionis, Vita Malchi captivi monachi, Vita sancti Pachumii, Vita sancte Eufraxie et aliorum patrum habentur in passionalibus ».

${ }^{62}$ Trèves, Stadtbibliothek, 1151/456 : Vita sancti Hilarionis (fol. 104r-113r); Vita sancti Malchi (fol. 113r-115v). 
Un autre membre de la communauté a joué un rôle central dans la mise par écrit d'œuvres consacrées aux saints à une époque plus tardive : Jean Scheckmann $(† 1531)^{63}$. Auteur prolifique, on lui doit plusieurs «Heiltumsbücher» allemands et latins, des sortes de petits guides imprimés pour pèlerins qui pullulèrent à Trèves à la suite de l'Inventio de la SainteTunique en 1512 pour faire la promotion de l'histoire et des reliques de la ville et de ses églises ${ }^{64}$. Il est aussi à l'origine de plusieurs textes hagiographiques consacrés aux saints patrons de l'abbaye repris dans un légendier copié entre 1513 et 1519 (Berlin, Staatsbibliothek Preußischer Kulturbesitz, theol. lat. fol. 729) et on le soupçonne également d'être l'auteur d'une série d'autres écrits ${ }^{65}$. À côté de la Bible géante, cet autre légendier constitue un témoignage supplémentaire de la production de manuscrits de bonne facture à Saint-Maximin dans le premier quart du XVI ${ }^{\mathrm{e}}$ siècle. Sans entrer dans les détails, ce volume peut être rapproché d'un manifeste politique destiné à protéger les intérêts de l'institution : dans un contexte marqué par la volonté d'attirer les pèlerins, il fallait à tout prix défendre sa position face aux autres établissements de la métropole, notamment l'abbaye voisine de Saint-Matthias qui souhaitait

\footnotetext{
${ }^{63}$ Relativement peu de choses nous sont connues de la vie de Jean Scheckmann. La biographie la plus « détaillée » se trouve dans Die Benediktinerabtei, vol. 2, p. 1244-1246 ainsi que chez F. BEZNER, Von der Liturgie, p. 161166. Voir également P. Diel, « Johannes Scheckmann von St. Maximin und seine Geschichte der Belagerung Triers durch Franz von Sickingen », Studien und Mitteilungen aus dem Benedictiner- und Cistercienserorden, 8 (1887), p. 348-357. Scheckmann est surtout connu pour avoir traduit en latin les Medulla Gestorum Trevirensium de l'évêque auxiliaire Jean Enen, activité qui est d'ailleurs consignée dans les comptes de l'abbaye pour les années $1518 / 19$ et 1519/20 (Trèves, Stadtbibliothek, 1626/401, p. 1211 et p. 1217). On suppose qu'il entra à l'abbaye de Saint-Maximin de Trèves au début du XVI $I^{\mathrm{e}}$ siècle et qu'il exerça le rôle de «bibliothécaire » (librarius) de cette institution. Dans une lettre de l'humaniste Jean de Borssele adressée à Scheckmann et reproduite dans l'Epitome alias medulla Gestorum Trevirorum - la traduction latine de l'œuvre d'Enen - le professeur de Louvain désigne explicitement son destinataire comme le responsable de la bibliothèque de Saint-Maximin («apud divum Maximinum bibliothecae curam agenti »). Toutefois, la fonction de «librarius » n'est pas autrement attestée à Saint-Maximin avant le XVIII ${ }^{\mathrm{e}}$ siècle. En 1524, deux ans après la destruction de Saint-Maximin par les habitants de Trèves, Scheckmann occupait l'office de coquinarius (Coblence, Landeshauptarchiv, Best. 211, n ${ }^{\circ} 1020$ ). Enfin, un obituaire-nécrologe inédit de l'abbaye nous apprend qu'il mourut le 12 août 1531: "Johannes Scekmannus sacerdos et monachus nostrae congregationis 1531 » (Trèves, Stadtbibliothek, 1635/48, fol. 8r-55v, cf. Die Benediktinerabtei, vol. 2, p. 1013-1014).

${ }^{64}$ La littérature entourant la « découverte » de la sainte Tunique dans la cathédrale est bien trop dense pour être répertoriée ici. Je me contenterai de renvoyer à l'ouvrage collectif Die Medulla gestorum Treverensium des Johann Enen : ein Trierer Heiltumsdruck von 1514, éd. M. ЕмBACH, W. ScHMID, Trèves, 2004 (Armarium Trevirense, 2), dans lequel certaines contributions évoquent les Heiltumsdrücke de Scheckmann. Les Heiltumsschriften de Trèves mériteraient une étude plus approfondie, particulièrement les livrets latins. Sur les Heiltumsschriften, cf. D. FEßL, Das spätmittelalterliche Heiltumsbuch als autonomer Publikationstypus - der erste Ausstellungskatalog neuzeitlicher Prägung mir Erinnerungswert, Thèse de Doctorat en Philosophie, Ludwig-Maximilians-Universität, Munich, 2013.

${ }^{65}$ Concernant le légendier, cf. J. P. BECKER et T. BRANDIS, Die theologischen lateinischen Handschriften in folio der Staatsbibliothek Preussischer Kulturbesitz Berlin, vol.2, Wiesbaden, 1985 (Kataloge der Handschriftenabteilung; Erste Reihe: Handschriften,2), p.283-285. Pour ce qui est des textes hagiographiques, on doit à Scheckmann une Vie de Basin (BHL 1028), saint patron de Saint-Maximin, ainsi qu'une Histoire et Élevation de l'archevêque Poppon de Trèves (BHL vacat) : A. PONCELET, « L'auteur de la vie de s. Basin évêque de Trèves ", Analecta Bollandiana, 31 (1912), p. 122-147. Il pourrait également être l'auteur d'une Vie de saint Maximin (BHL 5824g), d'une Histoire de saint Agrice (BHL 179d) et d'une Passion des martyrs de Trèves et de la légion thébaine (BHL vacat).
} 
se positionner comme le lieu de sépulture de l'évêque Agrice, l'un des saints patrons de Saint$\operatorname{Maximin}^{66}$.

Le tournant des $\mathrm{XV}^{\mathrm{e}}-\mathrm{XVI}^{\mathrm{e}}$ siècles fut donc marqué par une intense activité bibliothéconomique visant à réorganiser le fonds de livres de l'abbaye ainsi que par la production de plusieurs œuvres notables, fruit du travail de moines engagés dans le monde du livre et de l'écrit. L'intérêt marqué de certains auteurs pour l'hagiographie ne se limita toutefois pas aux nouvelles productions puisque l'abbaye possédait, dans sa bibliothèque, une collection de premier plan regorgeant de légendes consacrées aux saints les plus divers. Le grand légendier, à un certain degré, dut servir de recueil de matériaux de travail mais nécessitait, avant toute chose, d'être adapté pour répondre à de nouveaux besoins.

\section{Achèvement et réorganisation pragmatique du grand légendier}

Comme je l'ai déjà évoqué, le projet du grand légendier n'avait pas entièrement abouti au XIII ${ }^{\mathrm{e}}$ siècle. Peu de temps après se serait manifesté un nouvel acmé de l'édition hagiographique : les Legendae Novae connurent un grand succès, de même que les légendiers en langue vulgaire. Plus concis, ces nouveaux types de collection concurrencèrent les anciens modèles au point de mettre à mal l'édition de légendiers « traditionnels ${ }^{67}$. C'est en tout cas la ligne de fond qui avait été esquissée mais qui se voit aujourd'hui de plus en plus nuancée ${ }^{68}$,

\footnotetext{
${ }^{66}$ Outre la Vita sancti Agritii (BHL 178 ; fol. 35r-48v), on y trouve également plusieurs autres textes témoignant du culte particulier rendu au saint patron de l'abbaye et de l'archevêché. La première de ces pièces (fol. 49r) remonte à l'archevêque Thierry II de Wied (1212-1242), celui-là même qui avait favorisé l'abbaye dans la première moitié du XIII ${ }^{\mathrm{e}}$ siècle et qui y consacra quatre nouveaux autels en 1231 . Daté du 12 janvier 1239 , soit de la veille de la fête de saint Agrice, cet acte accorde quarante jours d'indulgence aux pèlerins et fait suite à une disposition identique prise par le pape Grégoire IX le 7 janvier 1236. Cette bulle pontificale est d'ailleurs reprise un peu plus loin dans le volume (fol. 51v). Entre les deux (fol. 49v-50v) se glisse un protocole relatif à l'ouverture de la tombe d'Agrice le 19 mai 1513 sous l'abbatiat de Thomas de Huisdem. Cette Inventio prend place dans le contexte des rivalités qui opposaient Saint-Maximin et Saint-Matthias concernant le lieu de sépulture de l'évêque. Le 12 juin 1378, sous l'abbé Rorich, le sarcophage d'Agrice avait été une première fois ouvert et on y avait trouvé une plaque de plomb portant le nom du saint. Cependant, en 1513, l'abbé de Saint-Matthias fit réaliser un reliquaire pour le bras d'Agrice et, le 7 mai, les moines découvrirent une pierre tombale avec le soi-disant cercueil de l'évêque. Pour faire face à cette « menace », l'abbé Thomas de Huisdem fit à nouveau ouvrir la sépulture d'Agrice, ordonna la mise par écrit de la découverte sur une dalle de schiste et fit consigner le tout par acte notarié. C'est précisément ce dernier document que l'on retrouve dans le légendier ainsi qu'une hymne au saint (fol. 51r) et l'Historia adventus sancti Agritii ad Treviros (fol. 52r-67r), texte qui pourrait être de Scheckmann. Dans le même état d'esprit, le légendier commémore également saint Thyrsus, saint Boniface et leurs compagnons (fol. 113r$118 \mathrm{v}$ ), soit les martyrs de la légion thébaine dont l'abbaye disputait la possession des corps aux autres établissements monastiques de Trèves dont Saint-Paulin J. P. BECKER et T. BRANDIS, Die theologischen lateinischen Handschriften, p. 284-285 ; Die Benediktinerabtei, vol. 1, p. 607-609.

${ }^{67}$ G. PHILIPPART, Les légendiers latins, p. 45-48.

${ }^{68}$ On pourra notamment renvoyer aux travaux suivants : V. VERMASSEN, «Latin hagiography in the Dutchspeaking parts of the Southern Low Countries (1350-1550)», dans Hagiographies. Histoire internationale de la littérature hagiographique latine et vernaculaire en Occident des origines à 1550, vol. VII, éd. M. GoULLET, Turnhout, 2017, p. 564-613 ; S. FOLKERTS, Voorbeeld op schrift : de overlevering en toe-eigenig van de vita van Christina Mirabilis in de late middeleeuwen, Hilversum, 2010 (Middeleeuwse studies en bronnen, 124).
} 
notamment dans le cadre de ce colloque ${ }^{69}$. Qu'en est-il des réalités tréviroises ? Je ne me risquerai pas à formuler d'hypothèses trop hâtives ${ }^{70}$, encore moins en me limitant au seul cas de Saint-Maximin dont le patrimoine hagiographique a été largement dispersé après la sécularisation (abstraction faite des pertes probables). Au XIV siècle, l'abbaye comptait parmi ses livres deux exemplaires de la Legenda aurea ${ }^{71}$. Au siècle suivant, elle se dota également d'une copie du légendier abrégé de Jean de Mailly ${ }^{72}$. C'est beaucoup moins que pour SaintMatthias, mais la conservation du fonds de cette abbaye est tout à fait exceptionnel et le nombre de légendiers « traditionnels » y demeure par ailleurs très largement majoritaire ${ }^{73}$. Aucune trace toutefois de légendiers allemands ${ }^{74}$, ni de livres imprimés de contenu hagiographique ${ }^{75}$. Les bréviaires, qui demeurent la terre en friche des études hagiographiques, sont plus nombreux

${ }^{69}$ Je renvoie à la communication de X. HeRmAND qui a très justement relevé pour l'espace namurois que « la composition [et l'édition] hagiographique tardo-médiévale [...] s'inscrivait encore dans les perspectives traditionnelles », avec d'une part la rédaction de textes neufs, mais aussi et surtout la compilation de collections, bien que cette activité eût été amoindrie.

${ }^{70}$ Ces questions sont au cœur du projet de recherche doctoral financé par le Fonds National de la Recherche luxembourgeois que je mène à l'Université du Luxembourg et à l'Université de Namur.

${ }^{71}$ Berlin, Staatsbibliothek Preußischer Kulturbesitz, lat. oct. fol. 223 et Trèves, Stadtbibliothek, 1173/37.

${ }^{72}$ Londres, Society of Antiquaries, 279.

${ }^{73}$ P. BeCKen, Die Benediktinerabtei, p. 76-240 ; Id., « Bibliothek der Abtei St. Matthias », dans Handbuch der historischen Bestände in Deutschland, éd. B. Fabian, vol. 6, Hildesheim, 1993, p. 245-248; J. MonTEBAuR, Studien zur Geschichte der Abtei St. Eucharius-Matthias zu Trier, Fribourg-en-Brisgau, 1931 (Römische Quartalschrift für christliche Altertumskunde und Kirchengeschichte. Supplementheft, 26). La majorité des manuscrits de l'abbaye ont par ailleurs été numérisés et sont consultables via le Virtuelles Skriptorium St. Matthias. Cf. M. EMBACH, «Das virtuelle Skriptorium St. Matthias: ein Projekt zur Digitalisierung mittelalterlicher Handschriften », dans Die Bibliothek der Abtei St. Matthias in Trier: von der mittelalterlichen Schreibstube zum virtuellen Skriptoirum, éd. M. EMBACH, Cl. Moulin, Trèves, 2013, p. 9-29.

${ }^{74}$ Pour les bénédictins, je n'ai relevé qu'un seul légendier d'apôtres provenant de Saint-Matthias (Trier, Stadtbibliothek, 812/1339). Plusieurs autres manuscrits hagiographiques vernaculaires proviennent quant à eux de l'abbaye de chanoines de saint Augustin d'Eberhardsklausen, notamment un légendier dédié à des saints locaux (Trèves, Stadtbibliothek, 809/1341) ainsi que la partie estivale de la Legenda aurea (Trèves, Stadtbibliothek, 1191/492) : B. C. BuSHEY, Die deutschen und niederländischen Handschriften der Stadtbibliothek Trier bis 1600, neu beschrieben, Wiesbaden, 1996 (Beschreibendes Verzeichnis der Handschriften der Stadtbibliothek zu Trier ; Neue Serie, 1); Die deutschen Handschriften der Stadtbibliothek zu Trier, éd. A. BECKER, Trèves, 1911 (Beschreibendes Verzeichnis der Handschriften der Stadtbibliothek zu Trier, 7). Sur les légendiers vernaculaires en langue allemande de manière générale, cf. W. WILLIAMS-KRAPP, Die deutschen und niederländischen Legendare des Mittelalters : Studien zu ihrer Überlieferungs-, Text- und Wirkungsgeschichte, Tübingen, 1986 (Texte und Textgeschichte, 20).

${ }^{75}$ L'exception serait un exemplaire des Dialogues de Grégoire le Grand de 1479/80 (Trèves, Stadtbibliothek, Inc. 1192). On relève à nouveau un nombre plus important de livres pour Saint-Matthias. E. VoulliEME, G. KENTENICH, Die Inkunabeln der öffentlichen Bibliothek und der kleineren Büchersammlungen der Stadt Trier, Leipzig, 1910 (Zentralblatt für Bibliothekswesen; Beiheft, 38), p. 89, n 1445. Je n'ai malheureusement pas encore eu l'occasion de consulter le nouveau catalogue : R. NOLDEN, Die Inkunabeln der Wissenschaftlichen Stadtbibliothek Trier, 2 vol., Wiesbaden, 2015 (Beschreibendes Verzeichnis der Handschriften der Stadtbibliothek zu Trier; Neue Serie, 3/1-2). Pour les autres fonds trévirois, cf. R. NOLDEN, « Die Inkunabeln in der Bibliothek der neuen St. Matthias-Abtei vor Trier », Kurtrierisches Jahrbuch, 50 (2010), p. 167-185; M. EMBACH, Die Inkunabeln der Trierer Dombibliothek: ein beschreibendes Verzeichnis mit einer Bestandgeschichte der Dombibliothek, Trèves, 1995 (Veröffentlichungen des Bistumsarchivs Trier, 29); Incunabula des Bischöflichen Priesterseminars Trier, éd. F. R. ReICHERT, M. EMBACH, L. BrinKHOFF, Wiesbaden, 1991. 
mais il s'agit souvent de copies personnelles de moines ${ }^{76}$. Ces nouvelles « armes » des cloîtres firent-elles définitivement basculer dans l'oubli les anciennes collections ? Certainement pas.

Un nouvel intérêt fut porté aux volumes du grand légendier dans la mesure où plusieurs mains pourvurent les textes inachevés d'initiales et d'intitulés. S'il s'avère difficile de situer ces interventions dans le temps ou de les attribuer à des intervenants précis, on peut affirmer qu'elles s'échelonnèrent sur une période chronologique assez longue et semblent plutôt avoir été le résultat d'actions individuelles que de véritables campagnes programmées.

On l'a vu, les volumes du grand légendier suivent grosso modo le déroulement de l'année liturgique, mais les incohérences sont nombreuses. Afin de pallier cette désorganisation accentuée par le caractère inachevé de la plupart des volumes, il était nécessaire d'en faciliter la consultation. C'est dans cette optique que l'on adjoignit des tables des matières aux manuscrits. À une exception près, celles-ci sont postérieures à l'époque de production du grand légendier et sont issues de mains différentes.

Seule la table du volume de mai (Trèves, Stadtbibliothek, 1151/454) pourrait être le reliquat d'un dispositif remontant à la genèse du grand légendier. Figurant en début de volume, la table des matières actuelle a été transcrite sur un texte préalablement gratté. Seul l'intitulé rubriqué en haut du feuillet permet de suggérer qu'il s'agissait déjà d'une sorte de table des matières organisée sous la forme d'un calendrier dont subsiste la colonne des lettres dominicales à droite (« Kalendarium [KL] : Majus mensis habet dies XXXI luna XXX. In hoc libro continentur passiones et actus sanctorum de mense Majo ») $)^{77}$.

Quant aux tables des volumes de septembre et d'octobre, elles peuvent être rattachées avec certitude à une seule et même main et débutent de la même manière :

- Trèves, Bibliothek des Bischöflichen Priesterseminars, 35 :

Horum sanctorum passiones vel vitas continet liber iste et secundum hunc ordinem eas invenies

- Trèves, Stadtbibliothek, 1151/456:

Horum sanctorum passiones vel vitas secundum hunc ordinem invenies in hoc libro

\footnotetext{
${ }^{76}$ Pour ne nommer qu'un seul exemple d'un bréviaire « de bonne facture »: Karlsruhe, Badische Landesbibliothek, aug. perg. 266 (XIV siècle). Il conviendrait d'étudier le lien entre les bréviaires et le patrimoine hagiographique d'une institution. À ma connaissance, G. PHILIPPART est l'un des seuls à avoir tenté l'expérience dans le cas d'un bréviaire monumental - certes beaucoup plus ancien - qui proviendrait de l'abbaye de Prémontrés de Knechtsteden : «Un bréviaire monumental de 1139 destiné aux prémontrés (?) de Knechtsteden (?) (Codex Bruxellensis 104)", dans Amicorum societas : mélanges offerts à François Dolbeau pour son $65^{e}$ anniversaire, éd. J. Elfassi, C. LANerY, A.-M. TurCAn-Verkerk, Florence, 2013 (Millennio medievale. Strumenti e studie, 34 ; Millenio medievale, 96), p. 601-634.

77 La plupart des volumes du grand légendier d'Autriche sont dotés de tables-calendriers. D. Ó RIAIN, « The magnum Legendarium Austriacum », p. 96. On en trouve aussi dans les deux légendiers susmentionnés de SaintMatthias de Trèves (Trèves, Stadtbibliothek, 1152/776 ; Bibliothek des Bischöflichen Pristerseminars, 5).
} 
Une particularité du support de la table du volume de février-mars-avril (Trèves, Stadtbibliothek, 1151/453) mérite d'être évoquée. Sur ce bifeuillet adjoint au manuscrit figure en effet un texte étranger au reste du grand légendier. Cette œuvre néo-latine versifiée s'intitule De prodigiis qui apparuerunt Rome in pontificatu Eugenii pape IV et serait l'œuvre d'un certain magister Porcellus. Il s'agit en fait d'un poème inédit du napolitain Porcelio de' Pandoni (ca. 1405-ca. 1485) : le Bos prodigiosus ${ }^{78}$. Sa présence dans le Treverensis avait jusqu'ici tout à fait échappé aux spécialistes ainsi qu'aux éditeurs de catalogues ${ }^{79}$. Le texte relate les «prodiges » qui se déroulèrent sous le pontificat d'Eugène IV, plus précisément à la mort de son prédécesseur, Martin V (1431), et à la suite de son élection sur le siège apostolique $^{80}$. Les circonstances dans lesquelles ce livret parvint à Trèves sont encore à élucider. Offert aux moines ou recopié par l'un d'entre eux, le bifeuillet véhiculant le poème fut ensuite remployé afin de servir de support à la table des matières du volume de janvier/février/mars. Sa présence à Saint-Maximin coïncide en tout cas avec la période marquée par un intérêt pour les textes humanistes (ca. 1450-1522) ainsi qu'avec l'abbatiat d'Antoine II de Drüblein (1452-1482) sous lequel de vastes opérations bibliothéconomiques furent initiées (cf. supra) $)^{81}$.

Initialement, la plupart des tables renvoyaient à une numérotation en chiffres romains des légendes. Pour assurer la correspondance, ces mêmes numéros ont été apposés de manière non systématique à côté de l'intitulé initial des légendes dans le corps des volumes. Toutefois, cette numérotation a majoritairement été grattée et remplacée ultérieurement par un renvoi à la foliotation des manuscrits ${ }^{82}$.

\footnotetext{
${ }^{78} \mathrm{Ce}$ texte a été très peu étudié et surtout mal interprété. Une description sommaire du contenu est donnée par U. Frittelli, Giannantonio de' Pandoni detto il Porcellio, Florence, 1900, p. 18-19. Depuis lors, l'historiographie s'est contentée de reprendre ce résumé sans revenir au texte. À propos de Porcelio de' Pandoni, cf. G. M. CAPPELLI, « Porcelio Pandone », dans Dizionario Biografico degli Italiani, vol. 80, Rome, 2014, p. 736740 ; A. IACONo, Porcelio de'Pandoni : L'umanista e i suoi mecenati. Momenti di storia e di poesia con un'Appendice di testi, Naples, 2017 (Latinae humanitatis itinera nova, 3), p. 43-57.

${ }^{79}$ Le poème n'était connu qu'à travers un unique manuscrit florentin (Biblioteca Nazionale Centrale, Conventi Soppressi, J IX 10, fol. 22v-26r). Sur ce manuscrit, cf. P. O. KRISTELler, Iter Italicum. A Finding List of Uncatalogued or Incompletely Catalogued Humanistic Manuscripts of the Renaissance in Italian and other Libraries, vol. 1, Londres/Leyde, 1963, p. 164.

${ }^{80}$ Les «prodiges » relatés se retrouvent en effet dans le troisième livre du De varietate fortunae de Poggio Bracciolini où ils se manifestent à la mort du souverain pontife et après l'élection de son successeur. Poggio Bracciolini, De varietate fortunae. Edizione critica con introduzione e commento, éd. O. MERISALO, Helsinki, 1993 (Annales Academiae Scientiarum Fennicae, Ser. B., 265).

${ }^{81}$ Précisons encore que c'est en 1472/1473 que fut créée l'Université de Trèves ce qui contribua également à ancrer la diffusion, l'étude et la rédaction d'œuvres humanistes dans la région: cf. M. EMBACH, Trierer Literaturgeschichte : Das Mittelalter, Trèves, 2007 (Geschichte und Kultur des Trierer Landes, 8).

${ }^{82}$ La numérotation des dossiers et les renvois à la foliotation sont des dispositifs tout à fait courant dans les manuscrits hagiographiques, particulièrement lorsque ceux-ci sont denses et complexes. Il n'est pas rare non plus que de tels dispositifs ne soient pas d'origine : F. DolBEAU, « Faire l'expertise de manuscrits ou de collections
} 
Dans les volumes de janvier, de février-mars-avril et d'octobre, les renvois à la foliotation sont attribuables à une seule et même main. Celle-ci a d'ailleurs intégralement rédigé la table des matières de février-mars-avril, ce qui permet donc de préciser qu'elle ne fut pas active avant l'arrivée à l'abbaye du bifeuillet véhiculant le livret contenant le poème de Porcelio de' Pandoni sur lequel elle fut retranscrite. On peut également affirmer que cette même main fut active avant l'ajout de la Vie de sainte Irmine d'Oeren qui a été copiée à cheval sur deux cahiers au sein du volume de janvier. En effet, le renvoi à ce texte fut ajouté a posteriori aux autres entrées de la table du volume de janvier. Notons encore que cette même main a rédigé la table des matières à la fin du volume des Causae et curae d'Hildegarde de Bingen, aujourd'hui conservé à la bibliothèque royale du Danemark ${ }^{83}$.

Outre le poème de Porcelio, d'autres textes, cette fois-ci relatifs aux saints, ont été copiés sur certains feuillets des volumes ou sur des feuillets adjacents. Une première addition textuelle se trouve dans le volume de mai (Trèves, Stadtbibliothek, 1151/454, fol. 125v) et concerne un miracle qui se serait produit à Saint-Maximin en 1444 lorsqu'une femme possédée recouvra l'esprit après s'être rendue au sous-sol de la crypte hors-œuvre dédiée à la Vierge ${ }^{84}$. Ce miracle eut lieu sous l'abbatiat de Lambert de Sassenhausen (1411/16-1449), l'un des successeurs de l'abbé Rorich $(† 1411)$ qui avait fondé une confrérie de sainte Marie (Marienbruderschaft) dans cette même crypte $^{85}$. C'est également à cet endroit, près d'un puits, que les moines de SaintMaximin firent apposer une inscription stipulant que saint Athanase d'Alexandrie, alors qu'il se cachait des Ariens, y avait découvert les reliques de martyrs de la légion thébaine, également évoquées dans le miracle de 1444 (in qua corpora trecentorum martyrum de gloriosa legione Thebeorum tumulata noscuntur) ${ }^{86}$. Or, cette précision n'a rien d'anodin. De fait, la présence des «300» martyrs de la légion thébaine à Saint-Maximin découle d'une tradition hagiographique complexe qui a synthétisé deux groupes de martyrs distincts, les martyrs de la légion thébaine d'une part et les citoyens trévirois massacrés par Rictiovare de l'autre ${ }^{87}$. Or, depuis le $\mathrm{XI}^{\mathrm{e}}$ siècle, Saint-Maximin et la collégiale de Saint-Paulin se disputaient le titre de lieu

\footnotetext{
hagiographiques », dans Ingenio facilis. Per Giovanni Orlandi (1938-2007), éd. P. CHIESA, A.-M. FAGNONI, R. E. Guglielmetti, Florence, 2017 (Millennio medievale, 111), p. 87-90.

${ }^{83}$ Copenhague, Det Kongelige Bibliotek, NKS 90 b 2 , p. 184-185.

${ }^{84}$ Le texte est édité dans M. CoENs, «Catalogus codicum hagiographicorum latinorum bibliothecae civitatis Treverensis », Analecta Bollandiana, 52 (1934), p. 199-200, n 38.

${ }^{85}$ Die Benediktinerabtei, vol. 1, p. 82. Concernant l'abbé, cf. vol. 2, p. 1080-1083. Le miracle est repris par Nicolas Novillanius, p. 1028. Ce dernier donne sa source : « Ex pervetusto libro anno Domini 1444, sub Frederico III ». À la fin de la transcription, il ajoute « Sub Lamberto abbate, post reformationem ».

${ }^{86}$ Die Benediktinerabtei, vol. 1, p. 616.

${ }^{87}$ Ibid., vol. 1, p. 612-617 ; K. KRÖNERT, L'exaltation de Trèves. Écriture hagiographique et passé historique de la métropole mosellane (VIII ${ }^{e}-X^{e}$ siècle), Ostfildern, 2010 (Beihefte der Francia, 70), p. 194 et 240-255.
} 
de sépulture de ces martyrs ${ }^{88}$. D'ailleurs, un autre témoignage de cette tradition manipulée se trouve sur le premier feuillet du même volume du grand légendier où l'on résume la découverte de reliques des saints martyrs dans un puits par saint Athanase d'Alexandrie, tout en précisant que cette découverte eut lieu à l'abbaye de Saint-Maximin de Trèves. Ces deux additions portées au volume de mai renseignent ainsi sur un aspect plus particulier du légendier comme support ou écrin de l'histoire et de la politique de défense de l'abbaye face à ses concurrents, notamment pour y attirer des pèlerins.

Une autre addition de premier plan concerne la Vita sanctae Irminae (BHL 44714472) ${ }^{89}$, une œuvre élaborée par le moine Thiofrid d'Echternach au $\mathrm{XI}^{\mathrm{e}}$ siècle ${ }^{90}$. Transcrite à cheval sur deux cahiers du volume de janvier (Paris, Bibliothèque nationale de France, lat. 9741, p. 139-140), elle forme un ajout qui, indirectement, livre certaines informations quant à la disparition du manuscrit véhiculant le sanctoral de décembre. Fêtée le 24 de ce mois, la sainte fit peut-être son entrée dans les calendriers de l'abbaye à la fin du Moyen Âge mais ne s'y maintint que temporairement ${ }^{91}$. Dès lors, elle ne faisait probablement pas partie du programme initial du volume de décembre et son intégration ultérieure au sein du « mauvais » manuscrit n'a logiquement pu avoir lieu qu'après la disparition du volume de décembre, soit après 1393 puisque ce dernier est encore mentionné dans l'inventaire dressé sous l'abbé Rorich («Item December in uno volumine $»)^{92}$.

\footnotetext{
${ }^{88} \mathrm{Je}$ rappelle également qu'une place fut donnée aux martyrs de la légion thébaine dans le légendier du début du $\mathrm{XVI}^{\mathrm{e}}$ siècle, précisément dans le contexte d'un afflux massif de pèlerins à Trèves.

${ }^{89}$ Sur Irmine, co-fondatrice d'Echternach, de la famille des Pippinides, cf. F. DE VRIENDT, «Irmine », dans Dictionnaire d'Histoire et de Géographie Ecclésiastiques, vol. 26, Turnhout, 1995, col. 49-51.

${ }^{90}$ W. KoHL, «Thiofrid (1110)», dans Biographisch-Bibliographisches Kirchenlexikon, vol. 11, Herzberg, 1996, col. 1233-1234.

${ }^{91}$ À partir du XIV ${ }^{\mathrm{e}}$ siècle d'après P. MIESGES, Der Trierer Festkalender. Seine Entwicklung und seine Verwendung zu Urkundendatierung. Ein Beitrag zur Heortologie und Chronologie des Mittelalters, Trèves, 1915, p. 112. Il conviendrait néanmoins de vérifier chaque calendrier utilisé par Miesges. De fait, celui-ci regroupe dans ses tableaux plusieurs calendriers sous un seul sigle : $\mathrm{M}_{3}$ regroupe également $\mathrm{M}_{4}$ tandis que $\mathrm{M}_{5}$ comprend aussi $\mathrm{M}_{6}$ et $\mathrm{M}_{7}$. Dans le cas de sainte Irmine justement, Miesges renseigne notamment le $\mathrm{M}_{5}$. Or, lorsque l'on se rapporte au calendrier de $\mathrm{M}_{7}$ (Trèves, Stadtbibliothek, 451/797, fol. 7v), le nom d'Irmine n'apparaît pas. Par ailleurs, on ne peut affirmer dans l'absolu que chaque calendrier employé provienne avec certitude de Saint-Maximin de Trèves sans investigation plus poussée. Les datations des calendriers données par Miesges doivent également être abordées avec précaution. À la fin du XVI ${ }^{e}$ siècle, le nom de la sainte n'apparaît plus dans les calendriers de deux manuscrits vraisemblablement transcrits par des moines pour leur usage privé (Trèves, Stadtbibliothek, 443/1908 et 479/1613), comme le suggère l'apposition dans le second du nom de Maximin Schram de Vianden (Frater Maximinus Schram Viennensis), prieur de 1583 à 1589. À son propos, cf. Die Benediktinerabtei, vol. 2, p. 11681169. À la cathédrale, la fête de la sainte aurait été célébrée dès le $\mathrm{X}^{\mathrm{e}}$ siècle. Elle est encore mentionnée dans un calendrier du XI ${ }^{\mathrm{e}}$ siècle provenant de la collégiale de Saint-Siméon. A. KURZEJA, Der älteste Liber ordinarius der Trierer Domkirche (London, Brit. Mus., Harley 2958, Anfang 14. Jh.). Ein Beitrag zur Liturgiegeschichte der deutschen Ortskirchen, Münster, 1970 (Liturgiewissenschaftliche Quellen und Forschungen, 52), p. 171-172.

${ }^{92} \mathrm{M}$. KEUfFER, « Bücherei und Bücherwesen » p. $54, \mathrm{n}^{\circ} 9$.
} 
Jusqu'ici, l'historiographie avait vaguement daté cette addition du $\mathrm{XV}^{\mathrm{e}}$ siècle $^{93}$. Néanmoins, l'expertise paléographique permet de déterminer que le renvoi au texte dans la table des matières fut ajouté après la rédaction de celle-ci sur l'un des feuillets du livret contenant le poème de Porcelio (cf. supra). En outre, l'examen de l'écriture, une gothique semihybride, permet d'identifier la main qui transcrivit la Vie comme étant celle qui rédigea également l'intégralité du légendier dédié aux saints patrons réalisé entre 1513 et 1519 (Berlin, Staatsbibliothek Preußischer Kulturbesitz, theol. lat. fol. 729) ${ }^{94}$. Or, rappelons que certains membres de la communauté avaient été envoyés à Echternach pour réformer l'abbaye et que, parmi eux, certains jouèrent ensuite un rôle dans le domaine du livre, dont les abbés Thomas de Huisdem et Vincent de Cochem, ce dernier étant également l'auteur des deux premiers volumes de la Bible géante de Saint-Maximin. Par ailleurs, une partie de la communauté trouva refuge à l'abbaye luxembourgeoise suite à la destruction de 1522, comme nous l'apprend l'Historia Excidii Sancti Maximini, une chronique contemporaine des faits qui est plus que probablement l'œuvre de Scheckmann et qui est transcrite au début et à la fin du troisième volume de la Bible géante ${ }^{95}$. L'une ou l'autre occasion dut permettre le transfert de la Vie d'Irmine, d'autant plus que des échanges de livres entre les deux institutions sont attestés ${ }^{96}$.

L'œuvre de Thiofrid avait été recopiée à la fin du XII siècle par Thierry d'Echternach dans le Liber aureus de l'abbaye ${ }^{97}$. Toutefois, ce n'est pas de ce dernier que l'exemplaire du volume de janvier dérive ${ }^{98}$. On peut supposer que les travaux de réorganisation de la

\footnotetext{
${ }^{93}$ O. SCHNEIDER, Erzbischof Hinkmar und die Folgen. Der vierhundertjährige Weg historischer Erinnerungsbilder von Reims und Trier, Berlin/New York, 2010 (Millenium-Studien), p. 178 ; M. KNICHEL, « Irmina von Oeren. Stationen eines Kultes », dans Studien zum Kanonissenstift, éd. I. CRUSIUS, Göttingen, 2001 (Veröffentlichungen des Max-Planck-Instituts für Geschichte, 167 ; Studien zur Germania Sacra, 24) p. 192 ; MGH SS rer. Merov., vol. 7, p. 645 ; B. KRUSCH, « Reise nach Frankreich», p. 620 ; F. AvRIL et C. RABEL, Manuscrits enluminés, vol. 1, p. 148 ; K. KRÖNERT, La construction du passé de la cité de Trèves : VIII ${ }^{e}-\mathrm{XI}^{e}$ siècles. Étude d'un corpus hagiographique, Thèse de doctorat en Histoire, vol. 2, Université de Paris X-Nanterre, 2003, p. 610.

${ }^{94}$ Le scribe est probablement Nicolas Dubgin qui a laissé son nom dans une note marginale au fol. $67 \mathrm{r}$ du légendier. À son propos, cf. Die Benediktinerabtei, vol. 2, p. 1166 et 1195.

95 F. BEZNER, Von der Liturgie zur Geschichte, p. 248, VIII, 13: "Eramus namque alii in monasterio Echternacensi (...)».

${ }^{96}$ Vincent de Cochem copia en 1511/1512 le martyrologe d'Usuard, la règle de saint Benoît et un obituaire à destination de l'abbaye luxembourgeoise (Luxembourg, Bibliothèque nationale du Luxembourg, 136). D'autres manuscrits écrits ou importés par des moines de Saint-Maximin incluent encore un bréviaire ainsi qu'un psautierhymnaire (Ibid., 7 et 10) : M. EMBACH, « Das Skriptorium der Abtei Echternach im Mittelalter. Eine Skizze », dans Kostbare Handschriften und Urkunden aus Echternach und Trier. Eine Ausstellung der Stadtbibliothek und des Stadtarchivs Trier, mit Leihgaben aus der Nationalbibliothek Luxemburg und dem Domschatz Trier, éd. M. EmBaCh, R. Nolden, Trèves, 2010 (Trierer Bibliotheken, 42), p. 147 ; T. Falmagne, Die Echternacher Handschriften

${ }^{97}$ Gotha, Forschungs- und Landesbibliothek, Memb. I. 71, fol. 23r-25r.

${ }^{98}$ En effet, Thierry avait ajouté le nom de la mère d'Irmine, Nanthilde, au texte (édité dans $M G H S S$, vol. 23, p. 48), ajout que l'on ne retrouve pas dans le volume de janvier du grand légendier. O. SCHNEIDER, Erzbischof Hinkmar p. 341-342, n. 706.
} 
bibliothèque epternacienne menés par les moines de Saint-Maximin permirent de mettre la main sur d'anciens manuscrits ou livrets et que c'est sur la base de l'un de ces derniers que la Vie fut copiée. Il ne s'agit d'ailleurs pas du seul matériau de Thiofrid qui est inclus dans des productions maximiniennes du premier quart $\mathrm{du} \mathrm{XVI}^{\mathrm{e}}$ siècle : le manuscrit des Legendae patronorum comprend également deux sermons du moine epternacien ${ }^{99}$. De son côté, Scheckmann a utilisé et a copié certains extraits du Flores Epytaphii Sanctorum ${ }^{100}$ - le seul traité médiéval sur le culte des saints et la vénération de leurs reliques à côté de celui de Guibert de Nogent (De sanctis et eorum pigneribus) - pour la composition de ses Heiltumsdrücke ${ }^{101}$.

Tenant compte de ces éléments et puisque la Vie d'Irmine a été copiée dans le volume de janvier, on peut supposer que le volume de décembre avait été détruit ou spolié au cours de l'une des destructions de l'abbaye en $1433 / 34$ ou en $1522^{102}$. Toutefois, il semble qu'il a bel et bien été pris en compte lors de l'inscription des cotes au tournant des $\mathrm{XV}^{\mathrm{e}}-\mathrm{XVI}^{\mathrm{e}}$ siècles. En effet, les volumes de janvier et de novembre portent respectivement les cotes « N 2 » et « N 9 », ce qui suggère donc que le volume de décembre correspondait à « $\mathrm{N} 1$ », ce qui serait impossible en cas d'une disparition du volume en 1433.

À côté de la Vie de sainte Irmine qui fut copiée dans le premier quart du XVI ${ }^{\mathrm{e}}$ siècle, un autre ajout textuel retient l'attention : la Vita sancti Malachie episcopi, retranscrite à la fin du volume d'octobre (Trèves, Stadtbibliothek, 1151/456, fol. 147v-164v). Si le début de ce texte remonte au XIII ${ }^{\mathrm{e}}$ siècle (fol. $147 \mathrm{v}-160 \mathrm{r}$ ), il était demeuré incomplet et fut complété grâce à un binion sur lequel on transcrivit la fin de l'œuvre (fol. 160v-164v). Or, le scribe de cette « suite » laissa en fin d'ouvrage une indication chronologique : «Anno domini 1545 in recompactione libri adjectum ». Cette main est par ailleurs identique à celle qui transcrivit 1'Historia Excidii Sancti Maximini de Scheckmann dans le troisième volume de la Bible géante finalisée par Jacob

\footnotetext{
${ }^{99}$ Berlin, Staatsbibliothek Preußischer Kulturbesitz, theol. lat. fol. 729, fol. 131v-135v. J. P. BECKER et T. BRANDIS, Die theologischen lateinischen Handschriften, vol. 2, p. 28. Les sermons sont édités dans la Patrologia Latina, vol. 157, col. 405-410.

${ }^{100} \mathrm{~L}$ 'œuvre est connue par trois manuscrits du XII ${ }^{\mathrm{e}}$ siècle dont un seul provient de Trèves (Bruxelles, Bibliothèque Royale, 10615/729). Ce codex issu de Saint-Matthias avait néanmoins été acquis par Nicolas de Cues avec une série d'autres manuscrits au $\mathrm{XV}^{\mathrm{e}}$ siècle. Les deux autres témoins proviennent d'Echternach (Trèves, Stadtbibliothek, 1378/103 et Gotha, Forschungs- und Landesbibliothek, Memb. I 70). M. C. FERRARI, « Lemmata sanctorum. Thiofrid d'Echternach et le discours sur les reliques au XII ${ }^{\mathrm{e}}$ siècle », Cahiers de civilisation médiévale, 151 (1995), p. 215-225 ; Theofridus Epternacensis, Flores Epytaphii Sanctorum, éd. M. C. FerRARI, Turnhout, 1996 (Corpus Christianorum. Continuatio mediaevalis, 133) et son complément de 1999.

${ }^{101}$ On peut, grâce à une comparaison automatique par ordinateur, identifier un extrait du traité dans le Tractatulus non tam ornatus quam devotus, in laudem sancte ecclesie Treverensis, Mayence, 1512, un livret de portée assez générale sur la ville de Trèves et ses reliques: B. Dubuisson, A Monk's Legacy. OCR, Lemmatization, and Computational Exploration of Some of the Works of Johannes Scheckmann of Trier (†1531), mémoire inédit de Master en Digital Humanitites, KU Leuven, 2019.

${ }^{102}$ Après l'élection d'Ulrich de Manderscheid au siège archiépiscopal, celui-ci fut contesté par l'abbaye ce qui le poussa à mener des expéditions vengeresses qui débutèrent à la fin de l'année 1433 : Die Benediktinerabtei, vol. 1, p. 309 .
} 
Gladbach en 1527. Il ne peut cependant pas s'agir de Scheckmann lui-même, puisque ce dernier était décédé en 1531.

Les textes dédiés à sainte Irmine et à saint Malachie d'Armagh ne sont pas les seuls ajouts qui remonteraient au premier quart du XVI $\mathrm{e}^{\mathrm{e}}$ siècle. À l'extrême fin du volume de janvier (Paris, Bibliothèque nationale de France, lat. 9741, p. 452-453), une main a écrit un texte bref véhiculant une série d'informations miraculeuses sur saint Marus, évêque de Trèves dont le corps reposait dans la crypte de l'église collégiale voisine de Saint-Paulin ${ }^{103}$. Saint Marus était célébré le 26 janvier et sa présence au sein des calendriers de l'abbaye est constante depuis le $\mathrm{X}^{\mathrm{e}}$ siècle. Toutefois, aucun texte consacré à ce saint ne nous est parvenu mis à part les informations véhiculées dans les Gesta Treverorum (ca. 1100) ${ }^{104}$. C'est justement de ces derniers que le premier paragraphe de l'opuscule, livrant un bref aperçu biographique sur l'évêque, s'inspire en partie ${ }^{105}$ :

BNF, lat. 9741, p. 452 :

Hac in tumba saxea conditur corpus sanctissimi Mari, ab Eucharie quadragesimi Trevirorum archiepiscopi, qui hoc sacrum melyti Paulini templum, a barbaris una cum diversis urbis Trevirorum vastationibus destructum, reparavit. (...)
Gesta Treverorum (MGH SS, vol. 8, p. 158) :

(...) Cirillum, Iamnerius, Iamnerium Emerus subsecutus, Marum post se constituit ; qui monasterium sancti Paulini, a barbaris cum praedictis urbis vastationibus desolatum, reparavit; ubi et ipse sepultus requiescit. (...)

L'écriture de cette annotation s'avère extrêmement proche de certaines des écritures figurant au sein des volumes de la Bible géante. Or, au moment même où les deux premiers volumes de la Bible de Saint-Maximin étaient en cours de confection, on procéda, en 1515, à l'ouverture de la tombe de saint Marus ${ }^{106}$.

Une cinquième addition dont l'écriture suggère qu'elle remonte au premier quart $\mathrm{du}$ $\mathrm{XVI}^{\mathrm{e}}$ siècle est l'épitaphe de l'abbé Jean I ${ }^{\mathrm{er}}(1034-1035 / 36)$ qui fut annotée dans la marge de

\footnotetext{
103 «Hic sequitur aliqua de sancto Maro ab Euchario XL. Treverensis archiepiscopo qui jacet post altare quod est ad australem partem chori ecclesiae Sancti Paulini archipraesulis Treverorum et martyris ». Le texte est édité dans les Acta Sanctorum, janvier, vol. 2, p. 730-731.

${ }^{104}$ MGH SS, vol. 8, p. 111-200. Cf. K. KRÖNERT, L'exaltation, p. 277-287.

${ }^{105}$ Comme l'a démontré une confrontation automatique des deux textes à l'aide de l'ordinateur (cf. n. 101), Scheckmann s'est inspiré du texte figurant dans le grand légendier ou de son archétype pour sa biographie de l'évêque dans l'Heiltumsdruck de Saint-Paulin. Éventuellement, c'est Scheckmann lui-même qui fit copier le texte dans le grand légendier. Peut-être l'avait-il découvert à Saint-Paulin au cours de ses recherches. En tout cas, il qualifie le miracle dont il est question de «recentius». Or, le saxon paralysé ayant recouvré l'usage de ses membres dont il est question pourrait être un certain Heinrich Duver $\beta$ de Brakel qui a visité l'abbaye vers 1515 et dont le nom figure dans le livre de confraternité de Saint-Paulin (Trèves, Stadtbibliothek, 1675/346, fol. 51r). Cf. . F.-J. HeYen, Das Stift St. Paulin vor Trier, Berlin/New York, 1972 (Germania Sacra; Neue Folge, 6 ; Das Erzbistum Trier, 1), p. 291, n. 2.

${ }^{106}$ Ibid., p. 290-291.
} 
pied de l'un des feuillets du volume de janvier (Paris, Bibliothèque nationale de France, lat. 9741, p. 357) ${ }^{107}$. Neveu de Poppon de Stavelot, Jean occupa brièvement l'abbatiat à la place de son oncle. Il était commémoré à Saint-Maximin par une pierre tombale sur laquelle était transcrite le texte reproduit dans le grand légendier. Jean est essentiellement connu à travers les informations véhiculées à son sujet dans la Vita sancti Popponis (BHL 6898) et c'est justement sur l'un des feuillets reprenant cette légende (p. 341-366) que fut copié cette épitaphe avec un renvoi à l'aide d'une croix au passage concernant la mort de Jean ${ }^{108}$.

Enfin, je signalerai encore, dans le même volume, l'ajout à la toute fin de la Vie de sainte Paule (BHL 6548 ; p. 370-385) d'un éloge que saint Jérôme lui aurait consacré et qui est considéré comme étant le principal complément à sa Vie ${ }^{109}$. S'agissant comme précédemment de la mise en relation d'une inscription funéraire avec la Vie du saint à laquelle elle se rapporte, l'écriture de cette annotation présente toujours de grandes similitudes avec les autres additions susmentionnées, ce qui permet de la dater de la même époque.

Pour terminer, il convient encore d'évoquer certaines manipulations des cahiers des volumes du grand légendier. Dans le volume d'octobre (Trèves, Stadtbibliothek, 1151/456), à la toute fin du binion ajouté en fin d'ouvrage pour compléter la Vie de Malachie d'Armagh, figure, comme je l'ai déjà mentionné, l'indication d'une recompactio, soit d'un « réassemblage » du volume en 1545 (« Anno Domini 1545 in recompactione libri adjectum »). Il ne s'agit pas de la seule annotation de ce type puisqu'à la toute fin du volume de septembre (Trèves, Bibliothek des Bischöflichen Priesterseminars, 35), une autre main a indiqué : "Anno Domini recompactas $1546 f[$ uerunt $]$ paginas ». Le terme de «compactio », s'il désigne un « assemblage », une « liaison » ou une « réunion de choses diverses qui forment un tout », est également l'un des termes employés pour désigner la mise en reliure d'un livre ou tout simplement sa reliure («libri compactio $»)^{110}$. Or, en 1548 , des travaux de reliure étaient justement en cours de réalisation puisque les volumes de la Bible géante, finalisés en 1527, ne furent pas reliés avant cette année-là ${ }^{111}$. À n'en pas douter, les reliures des volumes du grand légendier, marquées par trois siècles d'existence, avaient plus que certainement subi les affres

\footnotetext{
${ }^{107}$ L'épitaphe est reproduite dans J. N. Von HonTHeIM, Prodromus Historiae Trevirensis, vol. 2, p. 981.

${ }^{108}$ Pour l'édition de la vie, cf. MGH SS, vol. 11, p. 291-316. Le passage consacré à Jean se situe p. 309, n 23. Quant au renvoi à l'épitaphe, dans le légendier, il se situe au niveau de la phrase : «Res itaque non post modicum ex condicto viri Dei effectum invenit, vivendique finem eodem nimirum anno ipse dedit ».

${ }^{109}$ Cette épitaphe a bénéficié il y a quelques années d'une édition et d'une étude complète. A. CAIN, Jerome's Epitaph on Paula. A commentary on the Epitaphium sanctae Paulae, Oxford, 2013 (Oxford early Christian texts). ${ }^{110}$ Les anciens dictionnaires latin-français regorgent d'exemples de définition. Pour n'en citer que deux: P. MONET, « Relieure », dans Inventaire des deux langues, françoise et latine, assorti des plus utiles curiositez de l'un et de l'autre Idiome, Lyon, 1636, p. 746 ; J. F. BoINVILLIERS, "Reliure », dans Dictionnaire des commençants Français-Latin, composé sur le plan des meilleurs dictionnaires, $8^{e}$ éd., Paris, 1851, p. 389.

${ }^{111}$ F. BEZNER, Von der Liturgie zur Geschichte, p. 67.
} 
du temps et rendaient nécessaire une entreprise telle que la « recompactio », la « remise en reliure » donc, bien que des réfections aient déjà pu être menées antérieurement.

Une mise en reliure impliquait de détacher chaque cahier de la couture de l'ancienne reliure puis de les réassembler à nouveau. Certains soins étaient donc requis dans la mesure où le désordre pouvait facilement s'immiscer dans la succession des cahiers si ceux-ci n'étaient pas soigneusement organisés. De même, une fois détachés des nerfs de reliure, les feuillets d'un cahier étaient volants et il fallait donc faire attention à ne pas les dissocier ou à ne pas les mélanger. Pour éviter ce genre d'incident, les cahiers pouvaient recevoir des signatures ou des réclames ${ }^{112}$. Toutefois, force est de constater que ce type de dispositif n'est pas homogène à travers la collection et demeure marginal ${ }^{113}$. De plus, il s'avère difficile de les dater sans prendre d'autres indices en considération : l'exploitation des données codicologiques suggère que les volumes, ou du moins certains d'entre eux, furent l'objet d'au moins deux campagnes de réfection des reliures. Une première avant et une seconde après 1470, probablement dans les années $1540^{114}$. On sait que l'abbaye possédait aux $\mathrm{XV}^{\mathrm{e}}$ et $\mathrm{XVI}^{\mathrm{e}}$ siècles son propre atelier de reliure au sein duquel des manuscrits plus anciens furent dépecés pour en récupérer le parchemin afin de le remployer dans la confection de nouveaux ouvrages, notamment pour la consolidation des couvertures d'incunables et de livres imprimés. Les volumes du grand légendier constituent d'ailleurs en eux-mêmes des témoins de ce travail de récupération puisque le parchemin vierge a systématiquement été récupéré par découpe où il pouvait l'être, éventuellement lors de l'une de ces campagnes de réfection des reliures ${ }^{115}$.

\footnotetext{
112 Malgré les précautions, certains « incidents » étaient inévitables, en témoigne l'ordre compromis des cahiers véhiculant le dossier de Laurent de Rome dans le volume d'août (BNF, lat. 9742, p. 63-102). Face à ce désordre, on a pris soin de rectifier le tir en indiquant au lecteur par des notes marginales comment retrouver la suite logique des textes («[...] quaere post VIII folia » ou « [...] reverte VIII folia »).

${ }^{113}$ Trois volumes seulement contiennent des réclames ou une signature des cahiers, y compris le volume d'octobre où l'on trouve la mention de "recompactio». Les deux autres volumes sont ceux de janvier et d'août (BNF, lat. 9741 et 9742). On notera une particularité du volume de janvier dans lequel deux signatures des cahiers sont repérables dont une, plus récente, inscrite au début de chaque cahier à la pointe traçante en chiffres arabes. Celleci est pratiquement imperceptible mais confirme la perte du quatrième cahier dès l'époque médiévale ainsi que la disparition de deux textes pourtant référencés dans la table des matières : la Passio Secundi martyris et la Vita sancti Anthonini martyris. Les saints en question sont plus que probablement Second (dit «cultus Ameriae, Eugubii, Pergulae etc. ») et Antonin de Pamiers dont les dossiers se trouvent dans l'un des légendiers de SaintMatthias de Trèves avec lesquels le grand légendier est apparenté (BHL 7559 et 569). Cf. n. 13 et 19. Le fait que ces saints n'étaient pas célébrés au mois de janvier et que le dossier d'Antonin de Pamiers se retrouvait ailleurs dans la collection (Trèves, Bibliothek des Bischöflichen Priesterseminars, 35, fol. 7v-10v) expliquerait que le cahier ait été enlevé du volume de janvier.

${ }^{114}$ Il semblerait qu'ailleurs aussi, la période 1470-1530 fut propice aux opérations de reliure. Cf. G. LANOË, " L'apport de l'analyse des reliures à l'histoire des bibliothèques (1470-1530) », dans Le berceau du livre imprimé. Autour des incunables. Actes des 'Rencontres Marie Pellechet' (22-24 septembre 1997) et des Journées d'étude des 29 et 30 septembre 2005, éd. P. Aquilon, T. ClaERR, Turnhout, 2010 (Études Renaissantes, 5), p. 199-205.

${ }^{115}$ Die Benediktinerabtei, vol. 1, p. 154-155. Le fait que l'abbaye ait disposé de son propre atelier de reliure ne doit pas étonner : la reliure relevait en effet souvent de la responsabilité de l'acheteur et non pas du vendeur. SaintMaximin, en acquérant des livres imprimés tout en poursuivant la manufacture de manuscrits, avait intérêt à
} 


\section{Conclusions}

Trouvant son origine dans une campagne de production de manuscrits lancée dans le second quart du XIII ${ }^{\mathrm{e}}$ siècle, l'ambitieux grand légendier de Saint-Maximin de Trèves était demeuré inachevé à bien des égards. Alors que l'ensemble des textes avaient été copiés dans leur intégralité, à l'exception de celui dédié à saint Malachie d'Armagh, l'absence des intitulés relatifs à de nombreux dossiers devait compliquer la consultation de la collection dans le cadre des utilisations imputées traditionnellement aux légendiers ${ }^{116}$. Ainsi, un grand nombre des légendes véhiculées par les volumes échappait à une identification précise, dont certains dossiers pourtant dédiés à des saints importants pour l'abbaye ${ }^{117}$. Le côté peu pratique, voire encombrant de ces gros manuscrits joua certainement en leur défaveur : on les conserva à cause du coût financier qu'ils avaient représenté, peut-être aussi par souci de mémoire envers les morts - à l'instar du prieur Frédéric -, mais surtout pour leur valeur symbolique ${ }^{118}$.

Potentiellement concurrencée par les légendiers abrégés mais aussi par d'autres types de manuscrits liturgiques tels que les bréviaires ou les lectionnaires, la collection fut éventuellement reléguée dans un coin de l'abbaye, si bien qu'en 1393, lors de l'inventoriage des manuscrits à la bibliothèque, elle ne faisait pas partie des livres employés au chœur ${ }^{119}$. Toutefois, bien conscients de la richesse textuelle que représentaient ces neuf gros volumes, certains moines s'efforcèrent d'en atténuer les défauts et d'en rendre la consultation plus efficace par l'adjonction de tables des matières renvoyant à une numérotation des dossiers. Parmi ces intervenants figure notamment Louis de Ratingen, à qui l'on doit également un volume de Vitae Patrum rédigé en 1461 avec renvoi explicite au grand légendier. Pourtant, loin

\footnotetext{
disposer de son propre atelier de reliure. M. M. FOOT, « Some changes in binding structure and decoration during the first quarter of the sixteenth century ", dans La reliure médiévale. Pour une description normalisée. Actes du colloque international (Paris, 22-24 mai 2003) organisé par l'Institut de recherche et d'histoire des textes (CNRS), éd. G. LANOË, Turnhout, 2008 (Reliures médiévales des bibliothèques de France ; hors-série), p. 236.

${ }^{116}$ Lectures à l'office, au réfectoire, au chapitre, sur les lieux de travail ou en privé. G. PHILIPPART, Les légendiers, p. 112-117 ; F. DolbeAU, « Faire l'expertise », p. 76-77 et 90-91 ; Id., « À propos des lectures ».

117 On peut donner l'exemple de Nicet, l'un des saints patrons de Saint-Maximin, dans le volume d'octobre (Trèves, Stadtbibliothek, 1151/456, fol. 19r-22r).

118 Comme le fait remarquer D. Ó RIAIN, «The Magnum Legendarium Austriacum », p. 91, à propos du grand légendier d'Autriche: "A collection containing the Lives of over five hundred saints, which covers a period running from Antiquity to the second half of the twelfth century creates the impression of a shared and continuous Christian past and of a universal Church ».

${ }^{119}$ Y avait-elle seulement eu sa place avant l'extrême fin du Moyen Âge tel que le laisse entendre une note marginale dans le volume de novembre (cf. n. 32) ? Comme le fait remarquer Fr. DOLBEAU, « Faire l'expertise », p. 76 : «dans les grandes abbayes, on utilisait [en principe] au choeur des lectionnaires de l'office (...) et au réfectoire des légendiers », mais « dans la pratique, il semble qu'en beaucoup d'endroits des lectionnaires aient parfois servi au réfectoire et que des légendiers aient aussi servi au choeur et même, d'après certaines de leurs annotations marginales, ad collationem ». J'ajouterai qu'à l'instar des évolutions codicologiques des manuscrits hagiographiques, ces emplois étaient eux aussi susceptibles d'évoluer d'une génération de moines à l'autre.
} 
de satisfaire les besoins des moines, les volumes connurent d'autres mutations au tournant des $\mathrm{XV}^{\mathrm{e}}-\mathrm{XVI}^{\mathrm{e}}$ siècles lorsqu'une vaste campagne bibliothéconomique fut enclenchée : les manuscrits furent alors dotés d'une foliotation et l'on adapta les tables des matières existantes. Jusqu'à cette époque, la place des neuf volumes du grand légendier au sein du fonds de livres de l'abbaye demeurait relativement vague. L'inscription des cotes («N 1-9») permit expressément de les identifier comme les neuf «premiers» livres de la catégorie « hagiographie ».

Désormais, les volumes du grand légendier pouvaient être consultés de manière efficace et suscitèrent certainement l'intérêt de moines tels que Jean Scheckmann. Plusieurs mains du $\mathrm{XVI}^{\mathrm{e}}$ siècle ajoutèrent d'ailleurs l'un ou l'autre texte sur les feuillets vierges du légendier, dont la Vie de sainte Irmine ou les notes relatives à saint Marus. Dans le domaine du livre, le tournant des $\mathrm{XV}^{\mathrm{e}}-\mathrm{XVI}^{\mathrm{e}}$ siècles fut très fécond à Saint-Maximin de Trèves. Cet intérêt renouvelé pour les « antiquités » du fonds doit certainement être mis en relation avec la production d'œuvres manuscrites luxueuses sur parchemin, telles que la Bible géante ou encore le légendier de legendae patronorum qui s'insère dans le contexte plus large des ostensions de reliques et de la défense des intérêts privés de l'abbaye. Il ne s'agit d'ailleurs que de la partie émergée des écrits hagiographiques de cette époque dont une étude plus détaillée, tenant compte des supports plus anciens, dont le grand légendier, serait tout à fait intéressante du point de vue de la transmission du patrimoine hagiographique. En 1522, la catastrophe s'abattit sur SaintMaximin de Trèves : incendiée par les Trévirois après le départ du mercenaire François de Sickingen, l'abbaye fut pratiquement rasée. Bien que son patrimoine manuscrit fût préservé, c'est sans doute au cours de ces événements que le volume du grand légendier contenant le sanctoral de décembre fut perdu, raison pour laquelle il ne figure pas parmi les manuscrits employés par les Bollandistes au siècle suivant pour l'édition des AASS.

La collecte des données codicologiques et leur mise en contexte ont prouvé que l'analyse d'une collection manuscrite aussi ample que le grand légendier de Trèves ne pouvait se limiter à une approche centrée sur l'époque de production. Dans les siècles suivants, les initiatives développées dans le cadre de la gestion de la bibliothèque eurent des conséquences non-négligeables sur l'aspect actuel des volumes. La mise en place de systèmes organisationnels, tels que les cotes ou, dans un autre registre, les tables permettant une meilleure circulation au sein de la matière, témoigne de l'intérêt des moines pour ces manuscrits bien après leur confection. Ces derniers œuvrèrent à pourvoir les textes des intitulés et des initiales manquants, parfois en lien avec d'autres productions (Louis de Ratingen et ses Vitae Patrum), ou encore à inscrire certains compléments textuels véhiculant des données chronologiques et 
historiques. Parallèlement à ces transformations, la production de manuscrits (Bible géante et légendiers), ainsi que l'acquisition des premiers livres imprimés et leur mise en reliure, encouragea la récupération de parchemin et justifia certaines campagnes de réfection des reliures des codices. Les manipulations successives des volumes du grand légendier à la charnière des époques médiévale et moderne reflètent donc d'une part les aléas historiques que connut l'abbaye (destructions et reconstructions) tout en témoignant d'autre part des réponses apportées à des besoins propres et évolutifs. Plus largement, elles démontrent que les manuscrits hagiographiques anciens continuaient à susciter l'intérêt des communautés religieuses, ce que les évolutions spectaculaires de l'édition hagiographique tardive tendent parfois à masquer. 